\title{
Condensación textual lexicográfica: esbozo de una concepción integral ${ }^{1}$
}

\author{
Alberto Bustos Plaza \\ Universidad Carlos III \\ Herbert Ernst Wiegand \\ Universidad de Heidelberg
}

\section{INTRODUCCIÓN}

Por el momento carecemos de una subteoría integral de la condensación textual lexicográfica. El esbozo de una teoría de este tipo es relativamente complejo y en este artículo se ha acometido una considerable simplificación; rogamos expresamente que esto se tenga en cuenta. De lo contrario, hubiera resultado imposible presentar un panorama comprensible de, al menos, los aspectos fundamentales de la subteoría en su conjunto. El presente esbozo de una concepción integral de la condensación textual lexicográfica constituye una parte diferenciada de una teoría de los soportes textuales lexicográficos, que, a su vez, constituye un módulo de una teoría general de la lexicografía ( $c f$. Wiegand 1998a: "Introducción"). Nos referiremos aquí a diferentes secciones de esta teoría de los soportes textuales lexicográfi$\cos ^{2}$. Se presuponen unos conocimientos básicos de la teoría, pues por motivos de espacio no es posible explicar expresamente en su totalidad los términos y el contexto teórico empleados ${ }^{3}$.

1 Este artículo, especialmente adaptado para el lector de habla española, se basa en Wiegand (1998b, también 2000: 1454-1489). En comparación con la versión alemana, las explicaciones se basan en ejemplos tomados de diccionarios en lengua española. Asimismo, se ha revisado el texto y se han actualizado la terminología y la bibliografía. Le damos las gracias a Reinhold Werner por sus provechosos comentarios. La investigación de Alberto Bustos se ha financiado en parte con fondos del Ministerio de Ciencia y Tecnología (Plan Nacional de Investigación Científica, Desarrollo e Innovación Tecnológica), asignados al proyecto BFF2002-04575-C02-01.

$2 \quad C f$. Hausmann y Wiegand (1989); Kammerer y Wiegand (1999); Konerding y Wiegand (1995); Meyer y Wiegand (1999); Pan Zaiping y Wiegand (1995) y Wiegand (1983a, 1983b, 1984a, 1987, 1988a, 1988b, 1988c, 1989a, 1989b, 1990, 1991, 1994, 1995, 1996a, 1996b, 1996c, 1996d, 1997a, 1998a, 1999, 2000a, 2001, 2002a, 2002b, 2002c, 2004).

3 No obstante, a diferencia de la versión previa en alemán, hemos incluido algunas aclaraciones de cierta extensión, así como indicaciones sobre los trabajos mencionados en la nota 2 y otros. 


\section{RELEVANCIA DE UNA TEORÍA DE LA CONDENSACIÓN TEXTUAL}

Nos limitaremos aquí a los diccionarios impresos. Es sabido que, en estos, los textos de los artículos lexicográficos (principalmente), presentan ciertas propiedades características, lo que supone una textualización de contenidos proposicionales genuinamente lexicográfica. Menos conocido es que se puede dar cuenta como resultado de diferentes tipos de procesos de condensación textual de prácticamente todas las manifestaciones de propiedades que se pueden considerar genuinamente lexicográficas en los textos de los artículos. Las manifestaciones de dichas propiedades se pueden observar en los correspondientes ejemplares textuales. Por tanto, una teoría de la condensación lexicográfica permite explicar importantes aspectos de la textualización lexicográfica desde unos presupuestos y una perspectiva unificados. Esto resulta de interés para la investigación metalexicográfica por tres motivos principales.

En primer lugar, esto permite comparar con exactitud diccionarios con detalles textuales más o menos diferentes pero pertenecientes a un mismo tipo de diccionario, pues, atendiendo a determinadas características textuales, se puede calcular el grado de condensación textual en el estricto sentido (matemático) de la palabra (véanse algunas observaciones en la digresión de 6.1.).

En segundo lugar, dado que el grado de condensación textual influye considerablemente en la recepción textual del usuario concreto, la posibilidad metódica de calcular dicho grado representa un avance considerable en el desarrollo de una fórmula (disponible en principio para todo investigador) que permita calcular la facilidad de manejo de los diccionarios. Así, pasaríamos de afirmaciones más o menos fundadas sobre la facilidad de manejo (como las que se encuentran, por ejemplo, en numerosas recensiones sobre diccionarios) a afirmaciones con base científica, a las que, como mínimo, se les puede exigir que expliciten sus presupuestos teóricos.

En tercer y último lugar, una teoría de la condensación textual lexicográfica resulta de utilidad para presentar de forma comprensible la planificación de textos lexicográficos y, sobre todo, el diseño de los artículos lexicográficos, de manera que resulten identificables aspectos fundamentales del diseño de los textos lexicográficos (principalmente de los textos de los artículos), sobre todo los relacionados con la recepción de los artículos lexicográficos por parte del usuario concreto.

\section{PANORAMA DEL ESBOZO TEÓRICO}

La teoría de la condensación textual lexicográfica comprende las dos partes siguientes:

- subteoría de la condensación textual interna

- subteoría de la condensación textual externa 
La condensación textual interna tiene que ver con todas las entradas de diccionario accesivas ( $c f$. Wiegand 2004: 196) y, por tanto, con todos los textos lexicográficos parciales con portador de elemento conductor y/o con portador de dirección de remisión. Consecuentemente, la condensación textual interna tiene que ver sobre todo con aquellos artículos lexicográficos cuyo portador de elemento conductor es un lema de algún tipo ${ }^{4}$, así como con aquellas entradas de índice cuyo portador de elemento conductor es el elemento de acceso al índice. Por lo que respecta a los artículos lexicográficos con orden alfabético inicial, se pueden distinguir tres estadios de condensación textual interna.

En los artículos de signos lemáticos considerados monosémicos (o sea, así interpretados por los lexicógrafos; por ejemplo, adi $_{1}$-adi $_{4}$, abajo), el proceso de condensación textual sólo alcanza el primer estadio, y los procedimientos de condensación textual tan sólo se aplican a los elementos del superconjunto de la microestructura concreta ( $c f$. Wiegand 1989c), es decir, a las indicaciones y, concretamente, a la forma de estas ( $c f .6 .1)$.

En el caso de los artículos de signos lemáticos considerados polisémicos, el proceso de condensación textual alcanza el primer estadio, a continuación, un segundo $\mathrm{y}$, para ciertos tipos de microestructuras, un tercero. En el marco del segundo y tercer estadios de condensación textual interna en artículos de diccionarios alfabéticos, los procedimientos de condensación textual no sólo se aplican a los elementos de los superconjuntos de las microestructuras sino también a las relaciones de la estructura de constituyentes textuales concreta, en especial a las de las microestructuras (cf. 6.2 y 6.3).

La condensación textual externa, de la que se ocupa la segunda parte de la teoría, afecta a todos los textos lexicográficos parciales con estructura de acceso externa, o sea, sobre todo al catálogo central de palabras. Esta opera sobre los elementos de los superconjuntos de macroestructuras concretas y estructuras de acceso externas concretas, es decir, sobre los portadores de elemento conductor, así como sobre la macroarquitectura del catálogo de palabras central ( $c f .7)$.

No está prevista en la teoría una parte específica para la condensación textual relacionada con la medioestructura ( $c f$. Wiegand 1996a y 2002c). Esto es así porque, en el caso de las medioestructuras concretas, los procesos de condensación textual sólo pueden actuar sobre los elementos y no sobre las relaciones. Sin embargo, casi todos los elementos del superconjunto de la medioestructura pertenecen también al correspondiente superconjunto de las macroestructuras o de las microestructuras. De ahí que la condensación textual que afecta a los elementos de la medioestructura se trate en las partes ya mencionadas de la teoría. No es necesario que la teoría se ocupe expresamente de los escasos elementos medioestructurales que son al

4 Sobre los diferentes tipos de lema, véase Wiegand (1983a) y Wolski (1989). 
mismo tiempo elementos de la estructura-marco textual ( $c f$. a propósito de esta, por ejemplo, Wiegand 1995, Kammerer y Wiegand 1998) — como tampoco lo es para los otros resultados de la condensación textual que se manifiestan en la estructuramarco textual- El motivo es que no son resultados específicos de la condensación de textos lexicográficos, sino que suelen aparecer también en textos pertenecientes a otros géneros textuales 5 .

\section{CONCEPTOS BÁSICOS DE LA TEORÍA DE LA CONDENSACIÓN TEXTUAL LEXICOGRÁFICA}

La condensación textual, en consonancia con su relevancia para gran parte de los textos lexicográficos, desempeña también un papel fundamental en el sistema tipológico de los artículos lexicográficos en los diccionarios impresos ( $c f$. Wiegand 2004, 1999). En el marco de una tipología de los artículos basada en la condensación textual interna, se pueden diferenciar en primer lugar los artículos lexicográficos no condensados de los condensados. Los primeros son irrelevantes para el presente artículo. En cuanto a los segundos, podemos distinguir entre artículos total y parcialmente condensados. El criterio de exhaustividad permite decidir si un artículo lexicográfico está totalmente condensado. Este consiste en la presencia o ausencia de una indicación con forma textual en un artículo lexicográfico. Una indicación con forma textual es una parte de un artículo lexicográfico que comprende como mínimo una oración. Su sintaxis permanece intacta, lo que supone que no se ha visto afectada por ninguna forma de condensación textual que opere sobre la sintaxis. Un artículo lexicográfico sin ninguna indicación con forma textual está totalmente condensado; uno que contenga al menos una indicación con forma textual está parcialmente condensado. En adelante nos limitaremos a los artículos lexicográficos totalmente condensados.

Todo artículo lexicográfico estandarizado - es decir, todo aquel elaborado siguiendo un conjunto de estándares lexicográficos ( $c f$. Wiegand 1997a) de condensación textual - se puede considerar un condensado bimembre resultante de la aplicación de procedimientos de condensación textual al correspondiente texto integro. Para ello se tiene en cuenta el código de indicadores microestructurales (o, para abreviar, indicadores estructurales), que forma parte del programa microestructural. El texto íntegro consta de un título y del cotexto de este. Mediante la condensación textual y la aplicación del código de indicadores microestructurales

5 Conviene resaltar que los fenómenos de condensación textual desempeñan un papel más bien modesto en la investigación sobre lingüística del texto. Un ejemplo característico lo constituye el índice analítico de la bibliografía de Kirsten Adamzik (1995), que carece de entradas para condensación textual, condensación o compresión textual. Wolski (1989a) ha recopilado observaciones sobre densidad textual, semántica, informativa, etc. en bibliografía no referida a los diccionarios. 
(por ejemplo, negrita para el lema), el título se convierte en lema; el correspondiente cotexto se convierte en un conjunto de indicaciones en orden lineal. Estas se refieren al signo lemático y, en su caso, a una o más formas del paradigma del signo lemático; por tanto, están direccionadas hacia la indicación sobre la forma del signo lemático o hacia otra indicación. En este último caso nos encontramos con un cambio de tema en el interior del artículo. Si al resultado de la condensación del cotexto se le aplica el código de indicadores estructurales tipográficos (por ejemplo, cursiva, negrita, etc.) y no tipográficos (por ejemplo, coma, punto y coma, paréntesis, recuadros, etc.), se obtiene el texto del artículo. Todo artículo lexicográfico que no presente un precomentario como primer constituyente textual inmediato se puede considerar como un texto que consta de dos condensados parciales inmediatamente consecutivos a los que se les ha aplicado el código de indicadores microestructurales ( $c f$. figura 1).

Artículos de diccionario (adi) con precomentario son, por ejemplo, los siguientes: $\operatorname{adi}_{1}:$

*AVERADAMENTE: ad. m. Con verdad y certeza.

El caso fue, según me he informado de persona muy calificada que lo supo y entendió averadamente.

\section{CANSINO.}

\section{[Pagés]}

$\operatorname{adi}_{2}$ : †ESTELA. (Añade). Esta ciudad se fundó en tiempo que el rey de Aragón don Sancho Ramírez ganó de los moros a Barbastro y a Bolea. Al principio fue un pueblo pequeño y al presente ciudad noble de Navarra. Mariana lib. 10, cap. 2 .

[Covarrubias-Suplemento]

En el primer artículo, el precomentario consiste en una indicación en forma de símbolo (un asterisco) direccionada hacia la derecha. La explicación se halla en las reglas de uso ${ }^{6}$. En el segundo artículo, se trata también de una indicación en forma de símbolo direccionada hacia la derecha (esta vez, una cruz) ${ }^{7}$. Como el propio término indica, el precomentario es siempre es un constituyente textual inmediato del

6 Pagés explica así la indicación en forma de símbolo: "Van señaladas con asterisco las palabras que no están en el Diccionario de la Academia Española, y aquellas cuyas definiciones se han corregido o ampliado" ("Reglas para el uso de este diccionario").

7 Covarrubias explica al inicio del Suplemento el significado de esta indicación en forma de símbolo: "Las dicciones que tienen estrellas son añadidas de nuevo; y las que tienen Cruz se an de continuar en las dicciones del Tesoro". 
texto del artículo en su conjunto. Los artículos lexicográficos con precomentario se pueden explicar considerando que al finalizar el proceso de condensación se produce una dislocación hacia la izquierda. Esta no solo provoca una relajación textual sino también que el constituyente textual dislocado resulte más fácil de localizar, que se convierta en una indicación próxima al lema que, tras la ejecución con éxito por parte del usuario concreto de una actividad de acceso (cf. Wiegand 1997a: 319-323), no es necesario buscar primero específicamente en el artículo, sino que se percibe inmediatamente. La dislocación hacia la izquierda (al precomentario) de un constituyente textual procedente de un comentario que sigue al comentario sobre la forma constituye un caso destacado de topicalización lexicográfica. Esta consiste en la dislocación hacia la izquierda, precediendo al lema, de un constituyente que no pertenece a la posición lemática. Este pasa a encabezar así el artículo lexicográfico.

El texto íntegro es una construcción teórica necesaria. Esto es así porque, cuando se dice, por ejemplo, que un artículo lexicográfico está condensado, una interpretación posible (que utilizaremos aquí) es que se presupone un proceso de tratamiento del texto por el cual un texto $T_{1}$ se condensa para dar lugar a otro texto $T_{2}$. Este texto presupuesto $T_{1}$ es uno de los posibles textos íntegros. Para cada artículo lexicográfico se pueden proponer diversos textos íntegros que se pueden construir a partir del artículo lexicográfico condensado. Lo siguiente es válido para todos los textos íntegros correspondientes a un artículo lexicográfico:

a) Deben ser idénticos en cuanto a sus contenidos proposicionales elementales, que tienen que ver con la forma del signo lemático o con la forma de otras expresiones tratadas lexicográficamente, y también tienen que ser idénticos en cuanto al número y la secuencia de dichos contenidos proposicionales.

b) Pueden ser diferentes en la elección de la expresión relacional ausente que media entre el lema y la indicación de paráfrasis de significado o indicaciones de sinonimia.

c) Pueden diferenciarse en la superficie textual.

Sea el siguiente artículo lexicográfico:

adi $_{3}$ : grillete $s . m$. (preferentemente en plural) Pieza semicircula metálica con pasadores y cadenas que se usaba para sujetar los pies de los presidiarios: Todavía se usan los grilletes en algunos países.

[Salamanca]

8 También se puede interpretar que se compara el texto que se considera condensado con otro que se considera no condensado o menos condensado, con lo que $x$ está condensado se lee como $x$ tiene un tamaño (en relación con las normas válidas para las propiedades de determinados objetos). Las expresiones en las que el predicado condensado se refiere a entidades cognitivas, como, por ejemplo, pensamientos, resultan inadecuadas, puesto que no se pueden comprobar. 
Para este artículo se pueden construir, por ejemplo, los tres textos íntegros (TI) siguientes (las cifras entre corchetes no forman parte del texto íntegro):

$\mathrm{TI}_{1}:$ [1] Artículo lexicográfico para grillete.

[2] La ortografía de grillete es |grillete|

[3] La forma del singular es grillete.

[4] Grillete es un sustantivo.

[5] Grillete es masculino.

[6] Grillete se utiliza preferentemente en plural.

[7] Grillete significa pieza semicircular metálica con pasadores y cadenas que se usaba para sujetar los pies de los presidiarios.

[8] Un ejemplo del uso de grillete es todavía se usan los grilletes en algunos paises.

$\mathrm{TI}_{2}$ : [1] Artículo lexicográfico para grillete.

[2] El lema muestra la ortografía y la forma de singular.

[3] Grillete es un nombre masculino que se utiliza preferentemente en plural.

[4] Quiere decir pieza semicircular metálica con pasadores y cadenas que se usaba para sujetar los pies de los presidiarios.

[5] Se utiliza así, por ejemplo: todavía se usan los grilletes en algunos países.

$\mathrm{TI}_{3}:$ [1] Artículo lexicográfico para grillete.

[2] Grillete, cuya ortografía y forma singular aparecen reflejadas en el lema, es un sustantivo masculino utilizado (preferentemente en plural) para referirse a una pieza semicircular metálica con pasadores y cadenas que se usaba para sujetar los pies de los presidiarios.

[3] El uso de grillete se ejemplifica en la siguiente oración: todavía se usan los grilletes en algunos paises.

Es fácil darse cuenta de que los títulos ([1]), que forman parte ellos mismos del texto íntegro, son idénticos. En cambio, el cotexto de dichos títulos es diferente, de manera que los tres textos íntegros son diferentes si los consideramos en conjunto. Los cinco contenidos proposicionales elementales (p) constituyen aserciones metalingüísticas ( $c f$. Wiegand 1983a: 422 y ss.) sobre el formante de signo lemático de grillete y sobre las formas del correspondiente paradigma léxico del signo lemático. En los tres textos íntegros encontramos idénticos contenidos proposicionales, a saber:

$\mathrm{p}_{1}$ : que la grafía es $\mid$ grillete $\mid$

$\mathrm{p}_{2}$ : que tiene una forma singular grillete

$\mathrm{p}_{3}$ : que grillete pertenece a la categoría de nombre

$\mathrm{p}_{4}$ : que grillete es de género masculino

$\mathrm{p}_{5}$ : que el uso preferente es el del plural

Los predicados relacionales, en cambio, son diferentes:

- $\mathrm{TI}_{1}: \mathrm{x}$ significa $\mathrm{y}$ 
$-\mathrm{TI}_{2}: \mathrm{x}$ quiere decir $\mathrm{y}$

- $\mathrm{TI}_{3}: \mathrm{x}$ se utiliza para referirse a y

También es diferente la textualización escrita de los contenidos proposicionales $\mathrm{p}_{1}$ a $\mathrm{p}_{5}$, que se presentan arriba en la forma canónica que...

Se pueden fijar estándares para la reconstrucción de los textos íntegros (cf. 5.). La utilización de textos íntegros estandarizados es necesaria, como veremos, para calcular el grado de condensación textual de un artículo lexicográfico de tal manera que sea comparable con el de otros artículos.

Un texto íntegro estandarizado al que se le ha aplicado al menos una operación de condensación textual (y como máximo una cantidad finita de estas) mantiene diversas relaciones con el condensado (es decir, con un texto condensado en cuanto resultado de la aplicación al texto íntegro de, como mínimo, una operación de condensación textual). Las cuatro relaciones siguientes son especialmente importantes:

- $\mathrm{x}$ es un texto íntegro estandarizado respecto del condensado $\mathrm{y}$

- $\mathrm{y}$ es un condensado procedente de un texto íntegro $\mathrm{x}$

- y es más denso proposicionalmente que $\mathrm{x}$

- $\mathrm{x}$ es menos denso proposicionalmente que $\mathrm{y}$

Las dos últimas relaciones pueden regir también para diferentes condensados de un mismo texto íntegro.

Las operaciones de condensación textual se denominan también métodos de condensación textual. Representan conjuntos de procedimientos que se han de aplicar ordenadamente (véase más abajo la caracterización informal del método acortar) y son las siguientes: acortar, abreviar, omitir, dislocar, sustituir, enlazar, resumir y combinar. Cada método presenta diversas variantes. Para las variantes de los métodos existen diversas denominaciones, a partir de las cuales no siempre resulta evidente que nos hallamos ante variantes de un mismo método de condensación textual. Por ejemplo, la formación de un nicho (cf. Wiegand 1989: 386 ss.) es una variante especial del método enlazar. La introducción de indicaciones en forma de símbolo es una variante particular de sustituir y el procedimiento interno de recorte de texto de documentación ( $c f$. Wiegand 1993) es una variante de omitir.

Las instrucciones de estandarización para la condensación textual son las que determinan cómo se han de aplicar en cada caso los métodos de condensación textual, así como cuáles de entre los constituyentes textuales de una entrada de diccionario accesiva se han de considerar resultados textuales de la aplicación de métodos de condensación textual. Dichas instrucciones forman parte del libro de instrucciones para la elaboración del diccionario. En la figura 1 se representa lo indicado hasta aquí a propósito de $\mathrm{adi}_{3}$.

Evidentemente, los textos íntegros estandarizados que se generen siguiendo un mismo patrón para diversos artículos lexicográficos con microestructuras jerárqui- 
cas concretas isomorfas ( $c f$. , por ejemplo, la figura 7 en Wiegand 1991: 385) y que, por tanto, son isomorfos-cl (es decir, isomorfos en cuanto al conjunto y la clase de las indicaciones) respecto de una misma microestructura jerárquica abstracta ( $c f$. Wiegand 1991: 374 ss.) presentan un mismo número y una misma secuencia de contenidos proposicionales del mismo tipo ${ }^{9}$. Dos contenidos proposicionales son del mismo tipo cuando corresponden a dos oraciones asertivas en cada una de las cuales se realiza una aserción sobre una manifestación de una misma propiedad.

Aclararemos las consideraciones anteriores con un ejemplo. Sean los dos artículos lexicográficos siguientes:

adi $_{5}$ : boto $s . m$. Bota alta que se usa para montar a caballo: En Salamanca hacían buenos botos camperos.

[Salamanca]

adi ${ }_{6}$ : grecismo $s . m$. Palabra o expresión griega usada en otra lengua: Muchos grecismos penetraron en el español a través del latín eclesiástico.

[Salamanca]

Las microestructuras jerárquicas concretas de estos artículos se presentan en la figura 2 junto con la microestructura jerárquica abstracta isomorfa-cl.

En el marco de una explicación de los conceptos básicos de la teoría de la condensación textual lexicográfica, se ha de atender también brevemente a los conceptos de cohesión y coherencia. Hasta donde tenemos conocimiento, la bibliografía más reciente sobre lingüística del texto no nos obliga a revisar la posición mantenida en Wiegand (1988b: 79 ss.), basada en Hellwig (1984a, 1984b). Recapitularemos esta aquí. Se diferencian dos tipos de situaciones textuales: cohesión y coherencia. Se entiende por cohesión el conjunto de relaciones sintagmáticas que se dan entre los segmentos de un texto. Una situación cohesiva surge cuando mediante un texto se introducen objetos de referencia (conceptos de referencia) y a continuación se retoman estos (por ejemplo, mediante relaciones anafóricas) y se enlazan unos con otros mediante predicados y otras relaciones, ya sea implícita o explícitamente. El plano de los contenidos proposicionales de un texto, así como el plano referencial que lo acompaña, pertenecen a la cohesión. Esto mismo es aplicable a las relaciones de contigüidad, que se pueden inferir a partir de un conocimiento presupuesto. El principio de construcción textual que da lugar a una situación textual cohesiva se denomina progresión referencial. En general, se puede decir que un texto -y, por tanto, también un texto genuinamente lexicográfico como, por ejem-

9 También las estructuras parciales de las microestructuras jerárquicas concretas pueden ser isomorfas entre sí e isomorfas-cl respecto de una estructura parcial jerárquica abstracta de una microestructura (denominada entonces estructura abstracta correspondiente). Se encuentran numerosos ejemplos para las estructuras nucleares izquierdas en Wiegand (1990: 45 y ss., $c f$. también Wiegand 1997). 


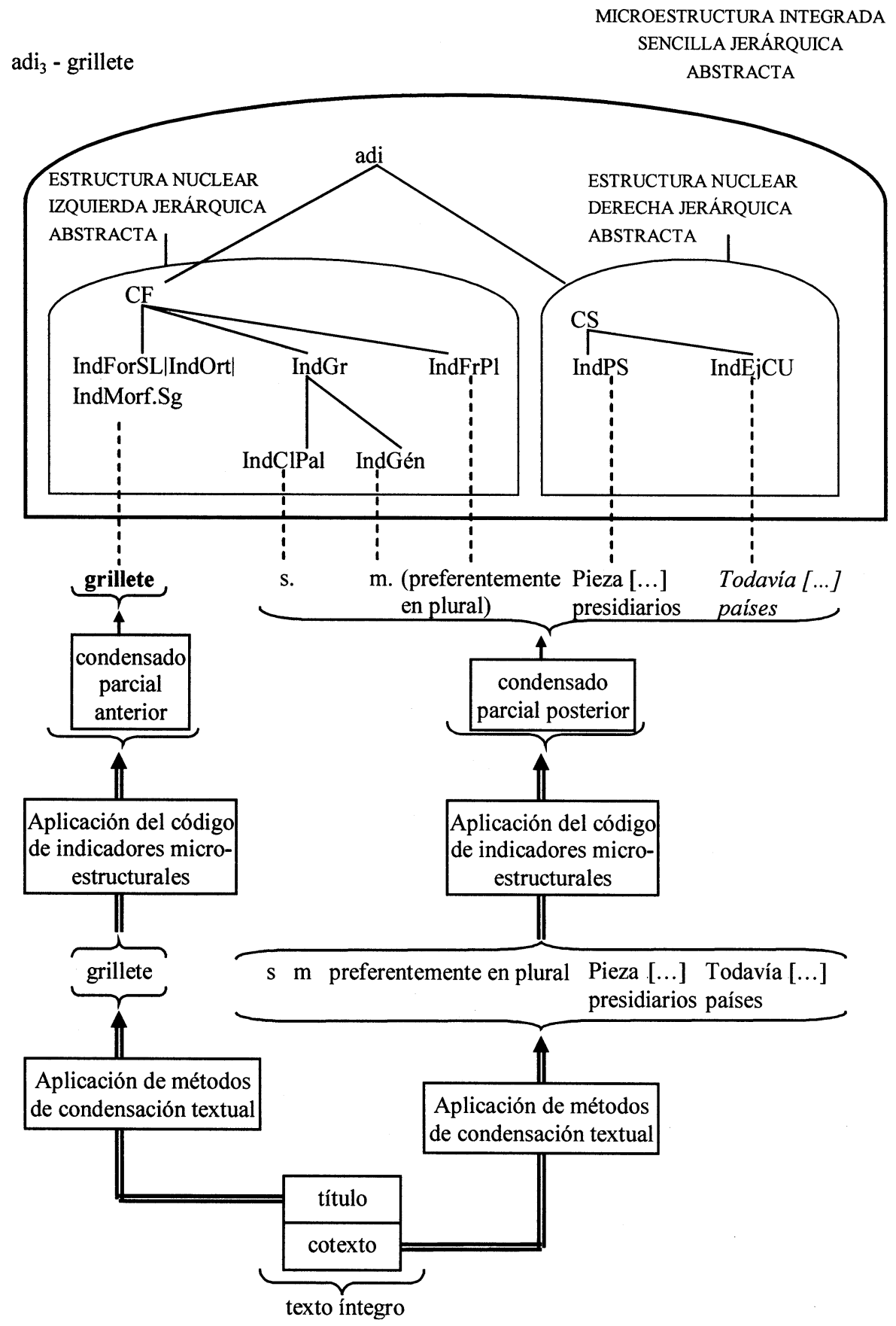

FIGURA 1. Representación de la condensación textual como paso de un texto integro a un condensado bimembre 
plo, un artículo lexicográfico- es cohesivo cuando las interpretaciones (en condiciones estándar) conducen a una relación textual en el plano sintáctico, proposicional y referencial. Un texto cohesivo no tiene por qué tener sentido, sino que llega a tenerlo cuando el texto se puede considerar coherente. Este es el caso cuando se puede llegar a una situación textual tal que de la sucesión sobre todo de oraciones pero también de otros segmentos textuales a los que se les pueden atribuir significados accionales (las denominadas ilocuciones) resulta un sentido; de modo que la coherencia -a diferencia de la cohesión- representa una genuina categoría pragmática que tiene que ver con la acción y comprensión sociales. Los temas textuales son fundamentales en la producción de la coherencia. El tema de un texto o de un fragmento textual es aquello de lo que se trata, sobre lo que se proporcionan explicaciones en el texto. La principal relación generadora de coherencia es la asignación de explicaciones a temas. Las sucesivas asignaciones de este tipo constituyen la progresión temática, que ha de considerarse el segundo principio de construcción textual ${ }^{10}$.

El concepto de cohesión es necesario para una teoría de la condensación textual lexicográfica, pues al aplicar métodos de condensación textual a un texto íntegro se pierden irreversiblemente partes del texto íntegro indicativas de la cohesión. Para que el usuario concreto pueda manejar con éxito el diccionario, debe disponer previamente (es decir, con anterioridad a la acción) del conocimiento generador de cohesión, que forma parte del conocimiento no especializado sobre la estructura de los artículos lexicográficos. Frecuentemente existen tradiciones que se reflejan en la condensación textual lexicográfica (por ejemplo, la omisión del predicado relacional entre el lema y la indicación de paráfrasis de significado) y que se corresponden con hábitos de recepción específicos de cada cultura en el marco de la práctica general de manejo del diccionario ( $c f$. Wiegand 1998a: 370). No obstante, como regla general se puede decir que los resultados de la condensación textual se han de explicar como mínimo en uno de los metatextos del diccionario (o en varios, como, por ejemplo, en las instrucciones de uso y en la lista de abreviaturas).

Disponemos ahora -aunque sea de manera informal- de algunos de los conceptos teóricos fundamentales. A continuación desarrollaremos la teoría de la condensación textual lexicográfica apoyándonos en ejemplos $\mathrm{y}$, por tanto, de manera eminentemente práctica.

10 Se puede ver un análisis de coherencia y cohesión para un artículo lexicográfico sencillo en Wiegand (1988b: 81 y ss.). 


\section{PATRONES DE ESTANDARIZACIÓN Y TEXTOS ÍNTEGROS ESTANDARIZADOS}

Para calcular el grado de condensación textual de los artículos lexicográficos es necesaria una estandarización. Estandarizar significa unificar a partir de un patrón, un esquema o un catálogo de instrucciones ( $c f$. Wiegand 1997a). En este apartado sentaremos en primer lugar las bases que permitan estandarizar el proceso de condensación textual u observar sus resultados a la luz de unos estándares.

Sea $\mathrm{adi}_{4}$, un artículo lexicográfico tomado del DUEAE:

$\operatorname{adi}_{4}$ : novación $n$. $f$. DER Sustitución de una obligación por otra otorgada, de modo que la primera queda anulada: la novación, compensación y remisión de la deuda extinguen la obligación, sin perjuicio de lo dispuesto en el artículo 1146.

$[D U E A E]$

A continuación construimos un texto íntegro estandarizado para adi ${ }_{4}$. Esto solo es posible si se dispone previamente de un patrón de estandarización. Por patrón de estandarización de textos íntegros se entiende un conjunto ordenado de esquemas asertivos (o formas asertivas) para oraciones asertivas metalingüísticas sobre las unidades tratadas en artículos lexicográficos pertenecientes a un determinado tipo de diccionario con cuyos lemas se mencionan signos lemáticos pertenecientes a un determinado tipo de signo lemático.

Para los fines demostrativos relativamente limitados de este apartado, nos conformaremos con constatar que el signo lemático novación pertenece a un tipo de signo lemático (TSL) que presenta el siguiente conjunto de propiedades constitutivas: \{sustantivo, monosémico, tratamiento monotemático\}. La última propiedad se refiere al hecho de que nos encontramos ante un artículo con direccionamiento lemático pleno (cf. Wiegand 1989b: 446) y, por tanto, ante un artículo lexicográfico sin cambio de tema, de modo que presenta un grado de complejidad relativamente escaso para su recepción por parte del usuario concreto. A efectos de la construcción de un patrón de estandarización, esto significa que nos basta en todas las formas asertivas con una única variable ("x") para las unidades tratadas lexicográficamente. A esta variable se le asigna un único ejemplar del correspondiente signo lemático al pasar del patrón de estandarización al texto íntegro estandarizado.

Nos serviremos con fines demostrativos del siguiente patrón de estandarización:

[1] Artículo lexicográfico para $x$.

[2] La ortografía de $x$ es $|. .$.$| .$

[3] La forma singular de $x$ es...

[4] La forma plural de $x$ es...

[5] $x$ es un nombre.

[6] $x$ es de género... [o...].

[7] $x$ carece de singular. 
[8] $x$ carece de plural.

[9] $x$ es contable.

[10] $x$ es incontable.

[11] La etimología de $x$ es... [o...], [IndEti].

[12] $x$ lleva la marca pragmática... [IndPrag].

[13] $x$ es pragmáticamente no marcado.

[14] $x$ significa... [o...], [IndPS o IndSin].

[15] $x$ tiene como sinónimo... [P1.].

[16] $x$ tiene como antónimo... [Pl.].

[17] Una colocación con $x$ es... [P1.].

[18] Un ejemplo del uso de $x$ es... [P1.].

[...] (otras formas asertivas, por ejemplo, sobre la pronunciación, la morfología, etc. pueden aparecer a continuación o introducirse en el lugar adecuado).

Este es un patrón de estandarización (no exhaustivo) para textos íntegros correspondientes a artículos lexicográficos para signos lemáticos de diccionarios generales monolingües pertenecientes al tipo de signo lemático $\mathrm{TSL}_{1}=\{$ sustantivo, monosémico, tratamiento monotemático $\}$. Antes de proseguir son necesarias algunas aclaraciones sobre el patrón:

a) Cuando aparece "o..." entre corchetes tras una forma asertiva, esto significa que la misma forma se puede emplear una o más veces y que las oraciones asertivas resultantes se han de enlazar mediante $o$, lo que da lugar a aserciones complejas sobre el contenido, tales que solo se ha de emplear una forma dada o una de las otras. Un ejemplo del doble empleo de la forma asertiva [6] es el siguiente: [6a. 6b.] azúcar es de género masculino o azúcar es de género femenino.

b) “P1." entre corchetes tras una fórmula asertiva indica que esta también se puede emplear en plural, con lo que se les tendrían que asignar al menos dos contenidos proposicionales a las oraciones asertivas resultantes.

c) Las abreviaturas de nombres de clases de indicaciones nos dicen a qué clase deben pertenecer las correspondientes indicaciones, que se pueden introducir en la posición reservada por el símbolo “...".

d) Al emplear el patrón de estandarización para generar el texto íntegro estandarizado correspondiente a un artículo lexicográfico, se incluyen en dicho texto íntegro las cifras entre corchetes de las formas asertivas, sin que dichas cifras se tengan en cuenta para la condensación. Cuando se emplea el procedimiento $o$, se cuenta alfanuméricamente (por ejemplo, 3a. 3b.). Si se emplea la forma plural, los contenidos proposicionales que se les asignan a las oraciones asertivas se cuentan de la siguiente manera: [15a/b/c] (por ejemplo, [15a/b/c] alegría tiene como sinónimos contento, regocijo, satisfacción). 
A continuación se le aplica el patrón de estandarización a adi ${ }_{4}$ con las correspondientes adiciones en sustitución de “ $x$ " $y$ “....". El resultado es el texto íntegro estandarizado $\mathrm{TIE}_{4}$ :

[1] Artículo lexicográfico para novación.

[2] La ortografía de novación es |novación|.

[3] La forma singular de novación es novación.

[5] Novación es un nombre.

[6] Novación es de género femenino.

[12] Novación lleva la marca pragmática derecho.

[14] Novación significa sustitución de una obligación por otra otorgada, de modo que la primera queda anulada.

[18] Un ejemplo del uso de novación es la novación, compensación y remisión de la deuda extinguen la obligación, sin perjuicio de lo dispuesto en el artículo 1146.

Como se puede ver en $\mathrm{TIE}_{4}$, al generar textos íntegros estandarizados solo se tienen en cuenta aquellas propiedades que han recibido tratamiento lexicográfico. De ahí que no se haya utilizado, por ejemplo, la forma asertiva [11] a pesar de que novación tiene una etimología; esta no se ha incluido en el correspondiente artículo lexicográfico, por lo que tampoco aparece en el texto íntegro estandarizado.

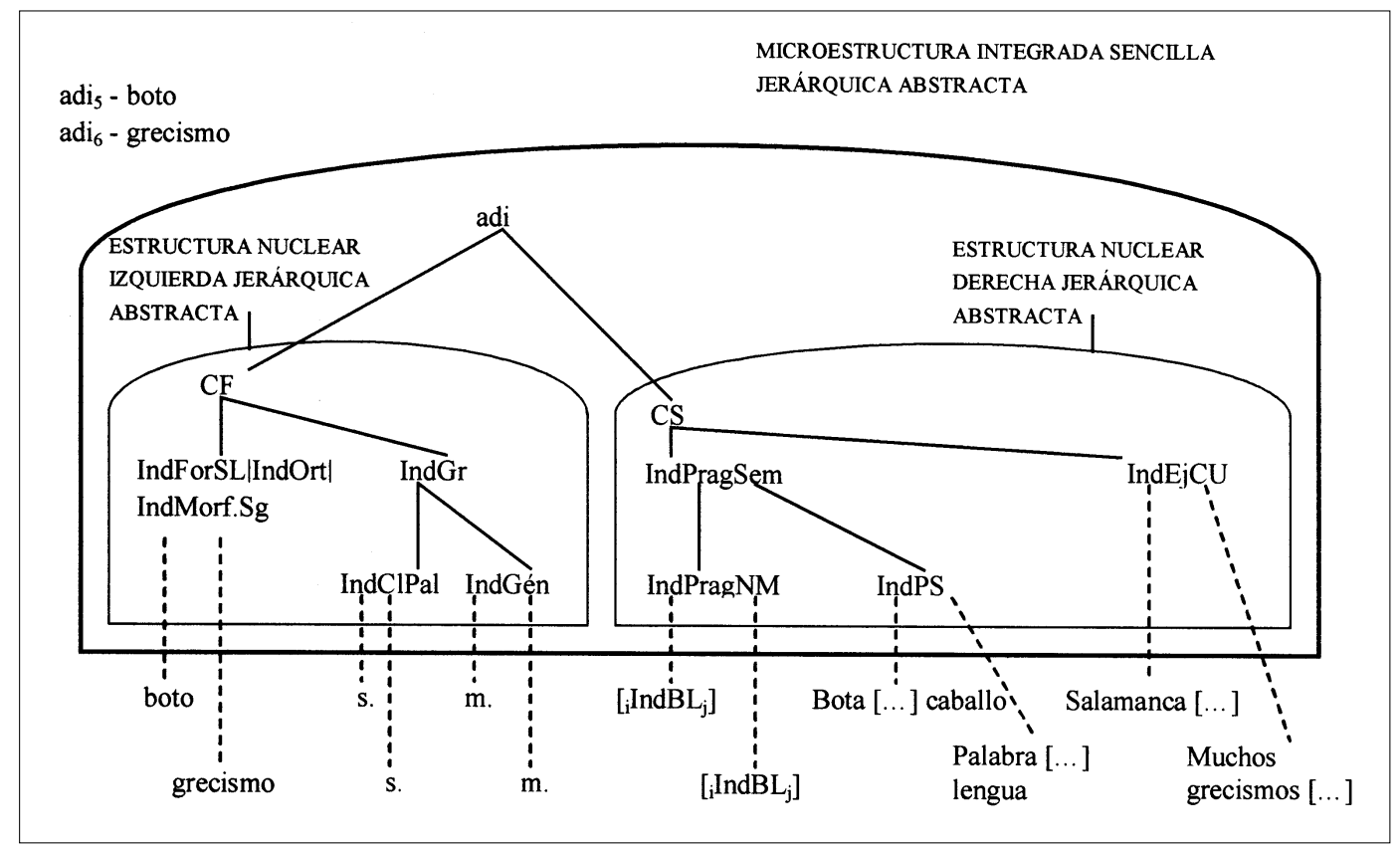

FIGURA 2. Diagrama estructural comentado de las microestructuras isomorfas que presentan los artículos lexicográficos adi $i_{5}$ adi 
Es fácil comprobar que los textos íntegros estandarizados para artículos lexicográficos con microestructuras isomorfas coinciden en el número, tipo y secuencia de los contenidos proposicionales. Para ello basta con construir los textos íntegros correspondientes a los dos artículos anteriores y contraponerlos:

$\mathrm{TIE}_{5}$

[1] Artículo lexicográfico para boto.

[2] La ortografía de boto es |boto|.

[3] La forma singular de boto es boto.

[5] Boto es un nombre.

[6] Boto es de género masculino.

[13] Boto es pragmáticamente no marcado.

[14] Boto significa bota alta que se usa para montar a caballo.

[18] Un ejemplo del uso de boto es en Salamanca hacían buenos botos camperos.
$\mathrm{TIE}_{6}$

[1] Artículo lexicográfico para grecismo.

[2] La ortografía de grecismo es |grecismo|.

[3] La forma singular de grecismo es grecismo.

[5] Grecismo es un nombre.

[6] Grecismo es de género masculino.

[13] Grecismo es pragmáticamente no marcado.

[14] Grecismo significa palabra o expresión griega usada en otra lengua.

[18] Un ejemplo del uso de grecismo es muchos grecismos penetraron en el español a través del latín eclesiático.

A partir de $\mathrm{TIE}_{5}$ se llega al condensado adi ${ }_{5}$ aplicando $n$ veces el método de condensación textual pertinente. Este proceso de condensación textual es idéntico al que lleva de $\mathrm{TIE}_{6}$ a adi $_{6}$ por lo que respecta a la aplicación $n$ veces de los métodos

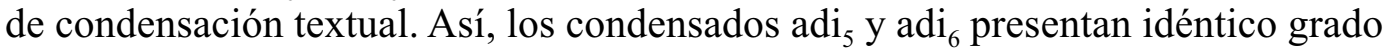
de condensación textual.

\section{CONDENSACIÓN TEXTUAL INTERNA}

La condensación textual interna afecta a las entradas de diccionario accesivas, como quedó de manifiesto en el apartado 3. A continuación examinaremos el primer estadio de condensación textual interna a propósito de adi $_{4}$.

\subsection{PRIMER ESTADIO DE CONDENSACIÓN TEXTUAL INTERNA}

Ya disponemos de un texto íntegro estandarizado para $\operatorname{adi}_{4}$ : $\mathrm{TIE}_{4}(c f .5$.). Fijémonos ahora en el título [1]. Se trata de un título descriptivo (cf. Hellwig 1984a). Estos presentan las tres formas de empleo siguientes:

(1) Son encabezamientos para un cotexto. Este es el caso en $\mathrm{TIE}_{4}$.

(2) Se emplean en un texto para referirse a otro texto (concretamente, a su cotexto). 
(3) Se utilizan en catálogos, bibliografías, etc. y también de forma abreviada en artículos lexicográficos para posibilitar la localización de los textos ${ }^{11}$.

Destacaremos aquí que, cuando se utilizan como encabezamiento de un cotexto, los títulos descriptivos tienen las seis propiedades siguientes, que no necesariamente se dan de manera simultánea en cada uno de ellos:

- Son breves metatextos del cotexto correspondiente.

- Contienen indicaciones sobre el objetivo pragmático del cotexto.

- Dan a entender de qué se trata en el cotexto y, por tanto, contienen indicaciones sobre el tema del texto.

- Pueden ayudar a interpretar el cotexto.

- Delimitan el cotexto externamente.

- Estructuran los textos en cuanto títulos de capítulo, intertítulos, títulos marginales, etc.

Si se le aplica al título del texto íntegro (o sea, "[1] Artículo lexicográfico para novación") el método de condensación textual acortar y, a continuación, el código de indicadores microestructurales tipográficos, se obtiene, como condensado parcial anterior, el lema novación.

Acortar como método de condensación textual se fija como sigue:

1. Ámbito de aplicación: cadenas lingüísticas que surgen de la aplicación de, como mínimo, una regla de encadenamiento sintagmático ${ }^{12}$.

2. Catálogo de procedimientos:

(I) Identificación de la cadena que se ha de acortar.

(II) Segmentación de la cadena identificando claramente las partes que se han de eliminar y las que no.

(III) Eliminación de las partes correspondientes.

(IV) Almacenamiento del resultado de la condensación para que quede disponible.

3. Control de aplicación: comprobar si el resultado de la aplicación del méto-

11 Su empleo en forma abreviada tiene lugar como indicación de inclusión en el diccionario, indicación de lugar de documentación o indicación bibliográfica. Estas indicaciones pertenecen a las siguientes medioestructuras intertextuales: de interconexión del diccionario, relativas a las fuentes y relativas a la bibliografia ( $c f$. Wiegand 1996a: 13 y ss.).

12 Esto supone que el método de acortamiento no es aplicable a las formas léxicas. Estas se abrevian (no se acortan). 
do se corresponde con el objetivo perseguido en este paso de la condensación textual ${ }^{13}$.

La cuestión es ahora cuáles de las seis propiedades atribuidas al título del texto íntegro presenta el condensado del título, es decir, el lema. Naturalmente, para contestar a las seis preguntas correspondientes, hay que tener en cuenta que un lema, en su aspecto genuinamente funcional como portador de elemento conductor, no se presenta separado ni aislado, sino solamente junto con otros lemas, formando parte de un diccionario.

La primera pregunta es la siguiente: ¿se pueden considerar los lemas como breves metatextos del correspondiente artículo lexicográfico? La respuesta es sí. Los motivos son los siguientes. En el título del texto íntegro se menciona el sustantivo novación. Tras eliminar la parte del título "Artículo lexicográfico para", nos queda una expresión mencionada que, tras aplicar el código de indicadores microestructurales tipográficos, se convierte en el lema. Por medio de este se menciona el signo lemático novación, que representa al paradigma del signo lemático, como unidad léxica del ámbito objeto del diccionario "español común actual". Esto significa que un usuario, a partir del lema, llega a saber algo sobre el correspondiente artículo lexicográfico, concretamente, qué parte del objeto del diccionario ( $c f$. Wiegand 1998a: 302) se trata en el artículo lexicográfico. La mención de signos lingüísticos es el único procedimiento lexicográfico posible para introducir el signo lemático en el artículo lexicográfico como objeto de referencia para posteriores predicaciones metalingüísticas que se presentan en forma condensada en las indicaciones direccionadas hacia el lema.

La respuesta a la segunda pregunta -si los lemas nos dicen algo sobre el objetivo pragmático del diccionario- es negativa. El título del diccionario es el único que nos dice algo al respecto.

La tercera pregunta -si el lema nos dice algo sobre el tema del texto- tiene una respuesta afirmativa. Aquí hay que tener en cuenta que un lema forma parte siempre de un diccionario, que pertenece a su vez a un tipo de diccionario. En nuestro ejemplo, novación es un lema de un diccionario monolingüe general. Este lema indica que determinadas propiedades del signo lemático, sobre todo su significado y su uso habitual, constituyen el tema del correspondiente artículo lexicográfico. Suponiendo que el lema novación es parte de una sinonimia acumulativa, dicho lema remite entonces a otro tema del correspondiente artículo lexicográfico. El tipo de diccionario, por tanto, desempeña un papel en la determinación del tema.

13 Los métodos de condensación textual abreviar, omitir, sustituir, enlazar, resumir y combinar se pueden definir siguiendo este mismo esquema, aunque no lo haremos en el presente trabajo. Para el método de la dislocación, que presenta diversas variantes, es necesario un esquema más complejo. 
La respuesta a la cuarta pregunta -si el lema proporciona ayuda para la interpretación del artículo lexicográfico- también ha de ser afirmativa. Por ejemplo, en un artículo como adi $_{7}$ el lema le permite al usuario experto sustituir la virgulilla del ejemplo de competencia de uso por la palabra adecuada y, por tanto, interpretar correctamente el ejemplo, que forma parte del artículo lexicográfico.

adi $_{7}$ : $\quad$ moi·sés $\mid$ moisés $\mid \boldsymbol{m} .{ }^{*}$ Cuna pequeña hecha de material flexible y que se puede llevar de un lado a otro: el padre llevaba al recién nacido en un $\sim$ de mimbre.

$[D E L E]$

Para terminar, la respuesta a las dos últimas preguntas también es afirmativa, pues resulta evidente sin necesidad de mayores explicaciones que los lemas delimitan artículos lexicográficos externamente y estructuran el catálogo de palabras como intertítulos. Esto significa también que lo delimitan de manera perceptible en el ejemplar textual.

A continuación nos ocupamos del cotexto situado en el interior de $\mathrm{TIE}_{4}$. En primer lugar nos fijaremos en las oraciones [2], [3], [5] y [6], que constituyen conjuntamente el cotexto y que, condensadas, dan lugar al comentario sobre la forma (CF). La representación estructural del comentario sobre la forma se puede ver en la figura 3.

El proceso de condensación, que lleva del cotexto de $\mathrm{TIE}_{4}$ al condensado parcial posterior, se describe a continuación paso a paso.

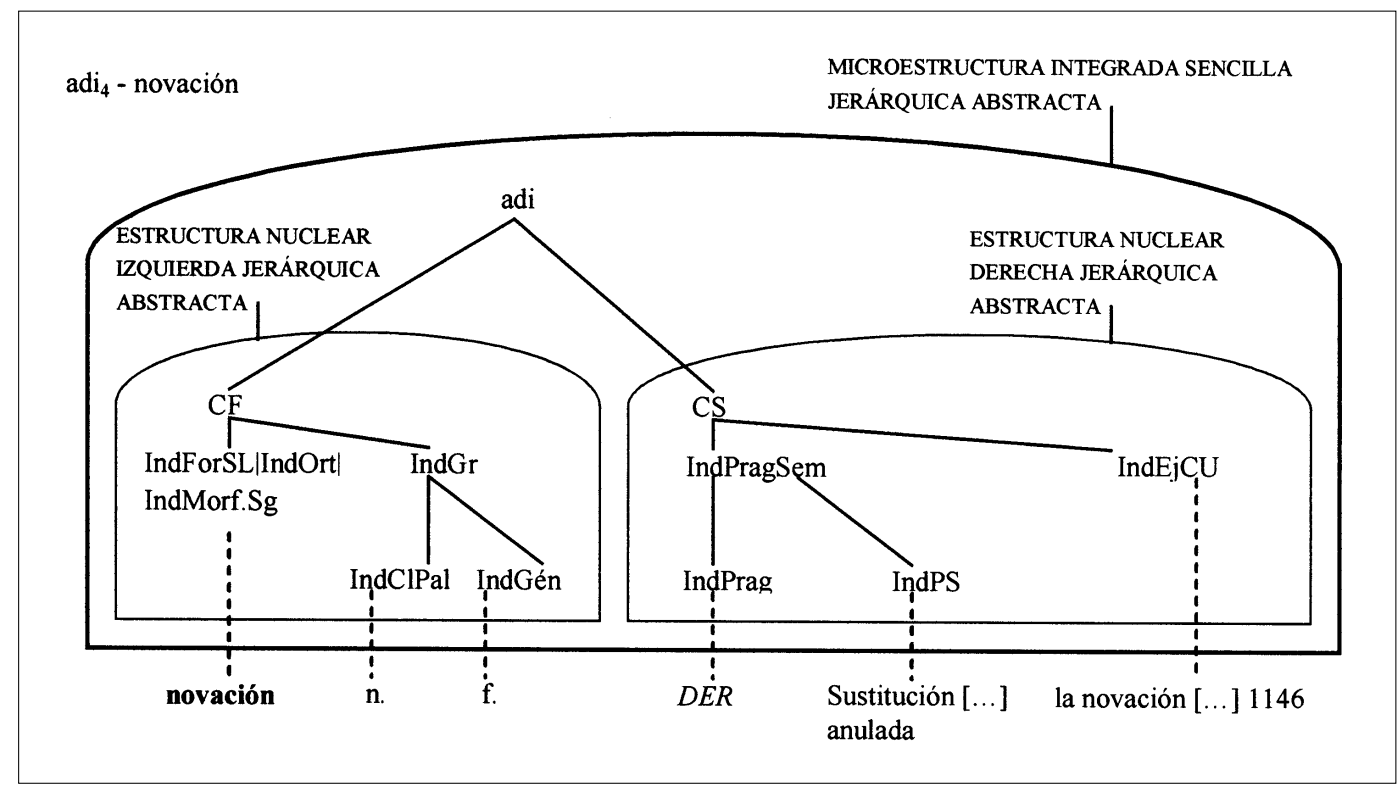

FIGURA 3. Diagrama estructural comentado de la microestructura jerárquica (concreta y abstracta) de adi ${ }_{4}$ 
Primer paso: la oración [2] del texto íntegro se omite $^{14}$. En su lugar, en uno de los metatextos del diccionario consta que la grafía de los lemas es ortográfica (o bien el usuario experto puede llegar a esa conclusión a partir de su conocimiento general de las convenciones lexicográficas). En la notación canónica que..., el contenido proposicional $\mathrm{p}_{2}$ correspondiente a [2] es el siguiente: $\mathrm{p}_{2}=$ que la ortografia de novación es |novación|. El usuario experto (cf. Wiegand 1998a: 506) adscribe $\mathrm{p}_{2}$ al lema novación. La indicación sobre la forma del signo lemático (IndForSL) es al mismo tiempo una indicación ortográfica (IndForSL|IndOrt, $c f$. figura 3 ).

Segundo paso: la oración [3] del texto íntegro se omite también. En su lugar, o bien consta en uno de los metatextos del diccionario que la forma canónica para los lemas de sustantivos es el singular, o bien el usuario experto puede llegar a esa conclusión a partir de su conocimiento general de las convenciones lexicográficas. El contenido proposicional correspondiente a la oración [3] es el siguiente: $\mathrm{p}_{3}=$ que la forma singular es "novación". El usuario experto también puede adscribir este contenido proposicional al lema. La indicación sobre la forma del signo lemático es, por tanto, simultáneamente, una indicación morfológica (IndMorf) para el singular (IndForSL|IndMorf.Sing, $c f$. figura 3).

Tercer paso: la oración [5] del texto íntegro se acorta suprimiendo "novación es un". La palabra restante nombre se abrevia en $n$ y se pospone al lema. Tras la aplicación del código de indicadores microestructurales, obtendremos una indicación de clase de palabras (IndClPal). El usuario experto le puede adscribir a esta indicación el contenido proposicional $\mathrm{p}_{5}=$ que novación es un nombre.

Cuarto paso: la oración [6] se acorta eliminando "novación es de género". La palabra restante femenino se abrevia en $f$, de forma que, una vez aplicado el código de indicadores microestructurales, tendremos una indicación de género (IndGén) a la que el usuario experto puede adscribirle el contenido proposicional $\mathrm{p}_{6}=$ que el género de novación es femenino.

Llegados aquí, es necesario aclarar expresamente que las indicaciones de un artículo lexicográfico se han de diferenciar claramente de la información lexicográfica que obtiene el usuario. La información lexicográfica consiste en entidades cognitivas. Estas no se obtienen únicamente a partir de una indicación. Más bien, el usuario concreto ha de referir la indicación a su correspondiente dirección, que siempre es otra indicación o una indicación parcial (en este caso, la indicación sobre la forma del signo lemático) y a continuación realizar inferencias. En otras pa-

14 Omitir, a diferencia de suprimir, es propiamente un método de condensación textual. Al aplicar el método de omisión se pierde siempre al menos un contenido proposicional, lo que no siempre es el caso cuando se produce una supresión como parte de métodos de condensación textual. 
labras: el usuario obtiene siempre la información lexicográfica a partir de un par ordenado de indicaciones o a partir de un par que consta de una indicación parcial $\mathrm{y}$ una indicación. Esto se logra mediante procesos de inferencia ( $c f$. Bläsi, Koch y Wiegand 1994: 231 y ss., Wiegand 1997a). Si, como en $\mathrm{adi}_{4}$, se da un direccionamiento lemático pleno, la primera coordenada del par ordenado es siempre la indicación sobre la forma del signo lemático.

Tras el cuarto paso, dentro del primer estadio de condensación textual, se les puede aplicar a los resultados del proceso de condensación textual el código de este diccionario para los indicadores microestructurales tipográficos y no tipográficos. Acto seguido, obtenemos el comentario sobre la forma $\left(\mathrm{CF}_{4}\right)$ de $\mathrm{adi}_{4}$, es decir, el siguiente texto parcial del artículo:

$\mathrm{CF}_{4} \mathrm{pd}^{15} \operatorname{adi}_{4}$ : novación $n . f$.

A continuación mostramos cómo las oraciones [12], [14] y [18] se condensan de tal forma que, al finalizar el proceso con la aplicación del código de indicadores microestructurales, obtenemos el comentario semántico (CS).

Quinto paso: la oración [12] se acorta eliminando "novación lleva la marca pragmática". La palabra restante derecho se abrevia en der. Tras aplicarle el código de indicadores microestructurales, que prescribe que las indicaciones pragmáticas aparezcan en cursiva y versalita, nos encontraremos ante una indicación pragmática sobre el uso de esta palabra en el campo del derecho.

Antes de que expliquemos el sexto paso, debemos referirnos a un fenómeno conocido, sobre el que se ha escrito abundantemente ( $c f$., por ejemplo, Wiegand 1985): en los artículos de los diccionarios modernos se suelen omitir los términos relacionales binarios que vinculan el signo lemático con la indicación de paráfrasis de significado. Este es también el caso en el DUEAE y, por tanto, en adi ${ }_{4}$. Nos limitaremos a mencionar de pasada que - incluyendo las posibilidades de las lenguas de especialidad - existen al menos 80 términos relacionales $(\mathrm{R})$ disponibles, entre los que se incluyen, por ejemplo, los siguientes:

$\mathrm{R}_{1}$ : un $\mathrm{X}$ es un $\mathrm{Y}$

$\mathrm{R}_{2}: \mathrm{X}$ significa $\mathrm{Y}$

$\mathrm{R}_{3}: \mathrm{X}$ quiere decir $\mathrm{Y}$

$\mathrm{R}_{4}$ : $\mathrm{X}$ se utiliza para referirse a $\mathrm{Y}$

En estos términos relacionales, " $\mathrm{X}$ " es una variable para signos lemáticos e "Y" para indicaciones de paráfrasis de significado. En el texto íntegro correspondiente a $\operatorname{adi}_{4}$ hemos escogido "X significa $Y$ ” para construir la oración [14].

15 La abreviatura $p d$ significa de aquí en adelante parte de. 
Sexto paso: a la oración [14] se le aplica el método acortar y se suprime el ejemplar del signo lemático novación, así como el ejemplar del término relacional significa. El resultado del acortamiento es sustitución de una obligación por otra otorgada, de modo que la primera queda anulada.

Séptimo paso: finalmente, se acorta [18] suprimiendo Un ejemplo del uso de novación es, con lo que nos queda la novación, compensación y remisión de la deuda extinguen la obligación, sin perjuicio de lo dispuesto en el artículo 1.146. Tras aplicar el código de indicadores microestructurales, que prescribe que los ejemplos de competencia de uso aparezcan en cursiva, se obtiene la indicación condensada de ejemplo de competencia.

Con el séptimo paso, se completa la transición de $\mathrm{TIE}_{4}$ a un condensado bimembre que, tras aplicar el código de indicadores microestructurales, se convierte en adi $_{4}$, perteneciente al DUEAE (véase figura 4).

Es posible agrupar en un conjunto todas las operaciones de condensación textual que se tienen que ejecutar al menos una vez (o, dicho de otra forma, todos los métodos de condensación textual que se tienen que emplear al menos una vez) para, a partir de un texto íntegro, llegar a un condensado bimembre que, tras aplicar el código de indicadores microestructurales, se convierte en un artículo lexicográfico para un signo lemático monosémico. La ejecución n veces $(\mathrm{n} \in N)$ (o, dicho de otro modo, la aplicación $n$ veces de los métodos de condensación textual de este conjunto) que es necesaria para obtener el condensado bimembre representa el primer estadio de condensación textual. Así pues, todos los artículos lexicográficos para signos lemáticos interpretados por el lexicógrafo como monosémicos se pueden entender como el resultado de procesos de condensación textual de primer estadio que tan solo se ven modificados ligeramente por la aplicación del código de indicadores microestructurales.

Digresión: consideraciones sobre el grado de condensación textual y su cálculo

El grado de condensación textual de los artículos lexicográficos puede variar. Por ejemplo, intuitivamente se percibe que adi' ${ }_{4}$ está menos condensado que adi ${ }_{4}$.

Adi' $_{4}$ : novación nombre femenino (propio del derecho) Sustitución de una obligación por otra otorgada, de modo que la primera queda anulada: la novación, compensación y remisión de la deuda extinguen la obligación, sin perjuicio de lo dispuesto en el artículo 1146.

De manera intuitiva, podemos decir que el grado de condensación textual de adi' ${ }_{4}$ es menor porque se trata de un ejemplar textual de mayor extensión al que se le pueden asignar los mismos contenidos proposicionales que al ejemplar textual más breve $\operatorname{adi}_{4}$. Evidentemente, la relación entre la extensión de un ejemplar textual y el número de contenidos proposicionales es fundamental en un juicio a partir 


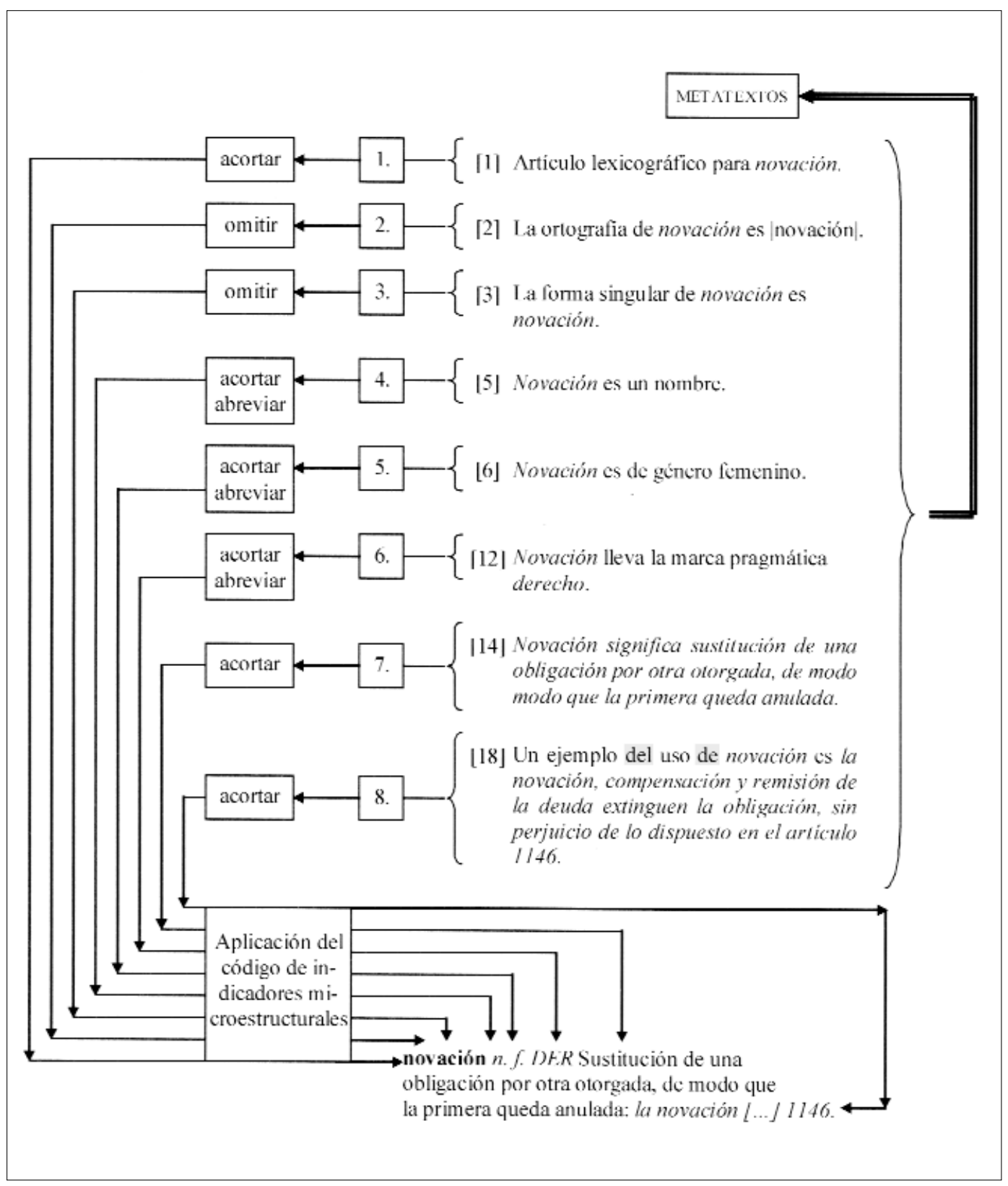

FIGURA 4. Representación de la ejecución metódica de un proceso de condensación textual que lleva de un texto íntegro estandarizado a un artículo lexicográfico e incluye la aplicación del código de indicadores microestructurales; las flechas con recuadros numerados significan Ejecución del paso $x$ aplicando el método correspondiente; la flecha " $f$ " indica que para cada operación de condensación textual realizada se ha de incluir una aclaración en uno de los metatextos del diccionario. 
del cual se llega a una constatación comparativa como la siguiente: adi $_{4}$ es proposicionalmente más denso que adi' ${ }_{4}$.

La cuestión ahora es cómo pasar de tales constataciones comparativas a constataciones métricas basadas en cálculos. Como dijimos en 5, a nuestro modo de ver, esto sólo es posible mediante estandarizaciones. A continuación presentamos algunas consideraciones sobre el cálculo del grado de condensación textual ${ }^{16}$ para las cuales nos serviremos de $\mathrm{CF}_{4}$ pd $\mathrm{adi}_{4} \mathrm{y}$ de las correspondientes oraciones [1], [2], [3], [5] y [6] de $\mathrm{TIE}_{4}$ :

\section{$\mathrm{CF}_{4}$ pd adi 4 : novación $n$. $f$.}

El conjunto ordenado de las oraciones [1], [2], [3], [5] y [6] constituye el texto íntegro estandarizado parcial $\mathrm{TIEP}_{4.1}$.

Para el cálculo necesitamos en primer lugar una unidad contable que nos permita expresar la longitud de los textos íntegros estandarizados. Aunque no podemos introducir aquí la pertinente justificación, emplearemos unidades contables a las que denominaremos ejemplares perceptibles de segmentos elementales de texto integro (segmentos elementales de texto integro para abreviar). Estas unidades de ejemplares de texto íntegro son las siguientes:

- todas las formas léxicas

— todos los signos de puntuación

- cualquier otro carácter que pueda aparecer en el interior del texto delante o detrás de una forma léxica (como, por ejemplo, paréntesis, barras verticales, etc.).

La oración [2] de $\mathrm{TIE}_{4}$ "La ortografía de novación es |novación|" consta, según lo anterior, de nueve segmentos elementales de texto íntegro. En consecuencia, tiene una longitud de 9. El texto íntegro parcial TIEP ${ }_{4.1}$ tiene, según esto, una longitud de 33. Como ya hemos mostrado, a este texto íntegro parcial se le pueden adscribir cuatro contenidos proposicionales elementales. Consecuentemente, el valor asertivo de $\mathrm{TIEP}_{4.1}$ es de 4 . Si calculamos ahora el cociente de la longitud y el valor asertivo de $\mathrm{TIEP}_{4.1}$, el resultado es $33: 4=8.25$. Este valor se puede tomar como medida de la densidad proposicional de TIEP $_{4.1}$. Cuanto mayor es el cociente, menor es la densidad proposicional de un texto íntegro (o de una parte de un texto íntegro). Lo interesante de la estandarización de un texto íntegro está, entre otras cosas, precisamente en que el cociente de los textos íntegros permanece constante, de manera que podemos calcular el grado de condensación textual del artículo lexicográfico en relación con este.

16 No podemos ocuparnos aquí de posibles alternativas a este proceso de cálculo del grado de condensación de textos lexicográficos. 
Para este cálculo son necesarias además unidades contables mediante las cuales se pueda expresar la longitud de los artículos lexicográficos plenamente condensados. A estas unidades se llega aplicando el método de segmentación funcional-posicional exhaustiva (método de segmentación (v) en Wiegand 1990: 22). El empleo de este método en $\mathrm{CF}_{4}$ pd $\mathrm{adi}_{4}$ da el resultado que representamos en la figura 5.

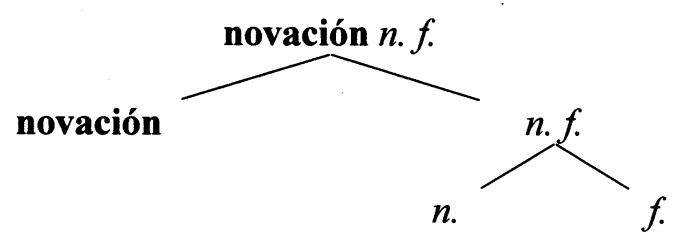

FiguRA 5. Resultado de la aplicación del método de segmentación funcionalposicional exhaustiva a $\mathrm{CF}_{4}$ pd adi $_{4}$.

Se cuentan los segmentos textuales funcionales terminales, es decir, las tres indicaciones "novación", " $n$." y " $f$.". Así pues, el comentario sobre la forma $\mathrm{CF}_{4}$ pd $\mathrm{adi}_{4}$ consta de 3 segmentos textuales funcionales, por lo que su longitud es de 3 . Como hemos visto, a $\mathrm{CF}_{4}$ pd $\mathrm{adi}_{4}$ se le pueden adscribir cuatro contenidos proposicionales elementales, por lo que el valor asertivo de este comentario sobre la forma es de 4 . El cálculo del cociente de la longitud y el valor asertivo nos da un valor para la densidad proposicional de $\mathrm{CF}_{4} \mathrm{pd} \mathrm{adi}_{4}$ de $3: 4=0.75$. Cabe preguntarse a continuación cuál sería el valor apropiado para el grado de condensación textual de $\mathrm{CF}_{4} \mathrm{pd} \mathrm{adi}_{4}$. Una posibilidad (entre otras) consiste en tomar la diferencia entre el cociente de la densidad proposicional de $\mathrm{TIEP}_{4.1}$ y el cociente de la densidad proposicional de $\mathrm{CF}_{4}$ pd $\mathrm{adi}_{4}$; es decir, $8.25-0.75=7.5$. Que esta diferencia sirve para expresar el grado de condensación textual queda de manifiesto si tenemos en cuenta que cuanto mayor es el cociente de la densidad proposicional del texto lexicográfico, menor es dicha densidad; el cociente aparece en la sustracción como sustraendo, de modo que el valor del grado de condensación textual es menor cuanto menor es la diferencia. De lo que aquí se trata es de calcular el grado de condensación textual, por lo que esta proporción (cuanto menor... menor...) es un resultado deseable. El grado de condensación textual de artículos comparables se puede comparar sistemáticamente mediante aserciones métricas en relación con el cociente constante para la densidad proposicional de un texto íntegro estandarizado.

No obstante, puede surgir un problema del que nos ocuparemos brevemente. Aplicamos a

$\mathrm{CF}_{4}{ }_{4}$ adi' $_{4}$ : novación nombre femenino

el método de segmentación funcional-posicional exhaustiva, lo que nos da el resultado que se representa en la figura 6 . 


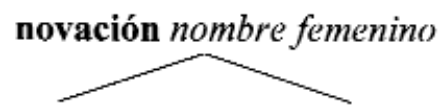

novación

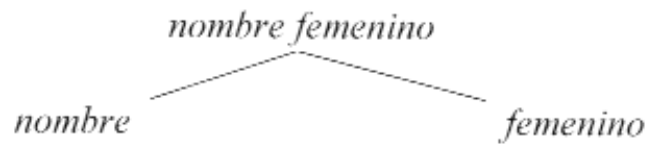

FIGURA 6. Resultado de la aplicación del método de segmentación funcionalposicional exhaustivo a $\mathrm{CF}^{\prime}{ }_{4} \mathrm{pd}$ adi $_{4}$.

Si comparamos ahora las figuras 5 y 6 , comprobaremos que el número de segmentos textuales terminales es el mismo. Naturalmente, este no es un resultado deseable, puesto que así obtenemos un mismo valor para el cociente de la densidad proposicional de $\mathrm{CF}_{4}$ pd $\mathrm{adi}_{4}$ y el de $\mathrm{CF}{ }_{4} \mathrm{pd} \mathrm{adi}_{4}$, con lo que el valor del grado de condensación textual es también el mismo. Sin embargo, esto contradice la intuición de que el grado de condensación textual de $\mathrm{CF}_{4}$ pd adi $_{4}$ es mayor que el de $\mathrm{CF}_{4}{ }_{4} \mathrm{pd}$ adi' ${ }_{4}$, puesto que en el primer caso se han abreviado nombre y femenino. Por tanto, se precisa un procedimiento - a ser posible, simple y general- que permita expresar mediante un cociente más elevado la menor densidad proposicional de $\mathrm{CF}_{4}{ }_{4} \mathrm{pd}_{\text {adi }}{ }_{4}$. Este procedimiento se puede describir como sigue: cada vez que se mencione con indicaciones constituidas por formas léxicas (como, por ejemplo, $f e-$ menino en $\mathrm{CF}_{4}{ }_{4} \mathrm{pd}$ adi $_{4}$ ) una forma léxica no abreviada o no acortada, esta indicación no condensada se cuenta dos veces. Así se eleva el cociente de la densidad proposicional de manera sistemática, es decir, homogénea para todos los casos comparables. Así se puede calcular fácilmente la menor densidad proposicional. Para nuestro ejemplo obtenemos los siguientes resultados:

$\mathrm{CF}_{4}$ pd adi ${ }_{4}$ : la longitud es ahora de 4, pues contamos dos veces la indicación sobre la forma del signo lemático, que aparece en forma no condensada ${ }^{17}$. Esta longitud se divide entre el valor asertivo (4), lo que nos da un cociente de 1. Este se le resta al cociente de la densidad proposicional de $\operatorname{TIEP}_{4.1}(8.25-1=7.25)$. Así tenemos 7.25 como valor del grado de condensación textual de $\mathrm{CF}_{4} \mathrm{pd} \mathrm{adi}_{4}$.

$\mathrm{CF}_{4}{ }_{4} \mathrm{pd} \mathrm{adi}_{4}$ : la longitud es en este caso de 6, por lo que el cociente de la densidad proposicional es de $6: 4=1.5$. Siguiendo el procedimiento anterior $(8.25-$ $1.5=6.75)$, obtenemos 6.75 como valor del grado de condensación textual. Como vemos, $6.75<7.25$, lo que da cuenta del menor grado de condensación textual de $\mathrm{CF}_{4}{ }_{4} \mathrm{pd} \mathrm{adi}{ }_{4}$.

17 En la versión alemana solo se contaba una vez la indicación sobre la forma del signo lemático. Hemos subsanado aquí este error. 


\subsection{SEgUNDO ESTADIO DE CONDENSACIÓN TEXTUAL}

En el caso de los artículos lexicográficos para signos lemáticos polisémicos, se han de tener en cuenta también las operaciones de dislocación hacia la izquierda (cf. Wiegand 1989a, 448 y ss.). Estas constituyen el segundo estadio de condensación textual interna. Operaciones de dislocación de este tipo dan lugar a estructuras textuales que, probablemente, son exclusivas de los textos lexicográficos.

Para aquellos textos representativos, objetivos, que no son artículos lexicográficos -y, por tanto, también para los textos íntegros-, se puede decir que las aserciones que aparecen en el interior del texto son válidas hasta que quedan canceladas por posteriores aserciones del mismo texto o hasta que se producen inferencias a partir de posteriores partes del texto que anulan su validez. Por tanto, las aserciones de un texto tienen campos de validez internos al texto y mantienen relaciones de validez, como, por ejemplo, si... entonces..., o... o..., etc. También las indicaciones de un artículo lexicográfico tienen campos de validez internos al artículo, lo que queda de manifiesto con especial claridad al considerar que proceden de las aserciones de un texto íntegro a las que se les han aplicado métodos de condensación textual. Que una indicación tiene validez lo indica, entre otras cosas, lo siguiente: para un par ordenado (constituido por una indicación y su dirección) hay como mínimo una aserción válida a partir de la cual se puede obtener el mismo contenido proposicional que a partir del par. También las indicaciones de los artículos lexicográficos mantienen relaciones de validez, y cuando estas no se corresponden con las de la constitución natural del texto, se deben regularizar de manera estandarizada, lo que se ha de explicar en uno de los metatextos. La aparición de relaciones de validez no naturales en artículos de signos lemáticos polisémicos se puede interpretar como determinada por la operación de la dislocación hacia la izquierda. Aclararemos esta a continuación a grandes rasgos.

Nos fijaremos para ello en uno de los casos más sencillos y frecuentes, a saber: la dislocación hacia la izquierda de indicaciones pragmáticas (IndPrag). Sea adi ${ }_{8}$, un artículo tomado del $D E A$ :

Adi $_{8}$ : corregimiento $m$ (hist) 1 Cargo de corregidor. | GNuño Madrid 49: El corregimiento de Madrid fue desem- peñado, bajo buena parte de su reinado, por un hombre ilustre: el Corregidor de Madrid .. don Francisco Antonio de Salcedo.

2 Territorio sometido a la jurisdicción del corregidor. | GHerrero Segovia 355: En el área de cada provincia persistían las antiguas y abigarradas subdivisiones: merindades, adelantamientos, corregimientos, hermandades, comunidades. Barcelona 53: En el año 1716 fue dispuesta por Felipe V, mediante el Decreto de Nueva Planta, la división de Cataluña en Corregimientos. 
En $\operatorname{adi}_{8}$, la indicación pragmática “(hist)" ('histórico') es válida para los dos subcomentarios semánticos, por lo que se ha dislocado hacia la izquierda, a la posición inmediatamente anterior a la indicación de polisemia "1".

En adi $_{9}$, en cambio, la indicación pragmática "coloq.", que afecta también a los dos subcomentarios semánticos, se repite en cada uno de estos:

Adi ${ }_{9}$ : pinchaúvas. $m$. coloq. Pillo que en los mercados comía la granuja, picándola con un alfiler, palillo u otro instrumento. || 2.coloq. Hombre despreciable.

[DRAE-2001]

En la figura 7, se encuentra el gráfico estructural comentado correspondiente al comentario semántico de $\operatorname{adi}_{8}$, y en la figura 8 el correspondiente al comentario semántico de adi $_{9}$.

Como se puede comprobar comparando los gráficos, por efecto de la dislocación hacia la izquierda aparece un nuevo constituyente inmediato en el comentario semántico: el subcomentario semántico dislocado hacia la izquierda. La correspondiente estructura parcial de la microestructura se denomina preintegrado. La dislocación hacia la izquierda altera la microestructura del artículo lexicográfico. La microestructura integrada simple se convierte en una microestructura simple parcialmente integrada con preintegrado. Consecuentemente, aumenta la densidad proposicional del texto lexicográfico, puesto que se suprimen las indicaciones

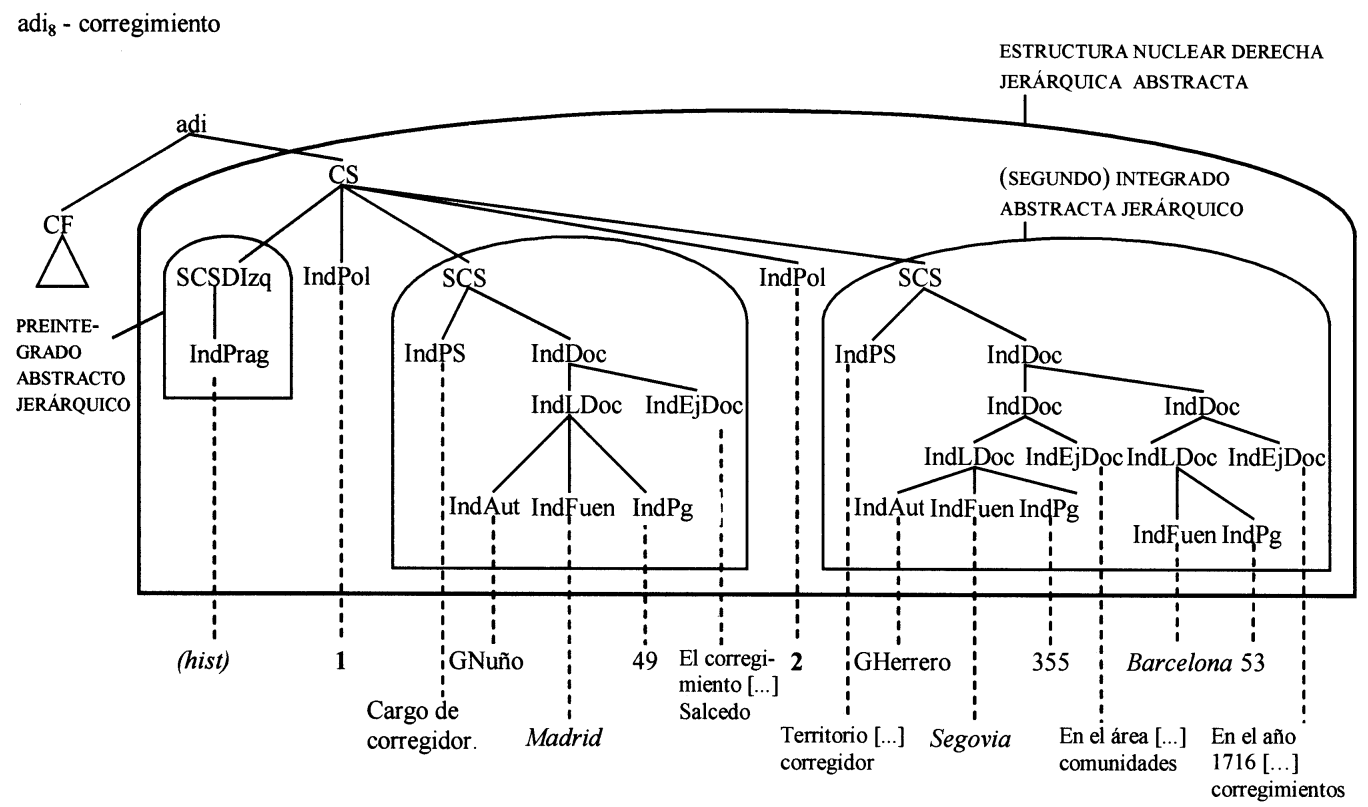

FiguRA 7. Diagrama estructural comentado de la estructura nuclear derecha jerárquica (concreta y abstracta) perteneciente al comentario semántico de adi ${ }_{8}$ 
adi $_{9}$ - pinchaúvas

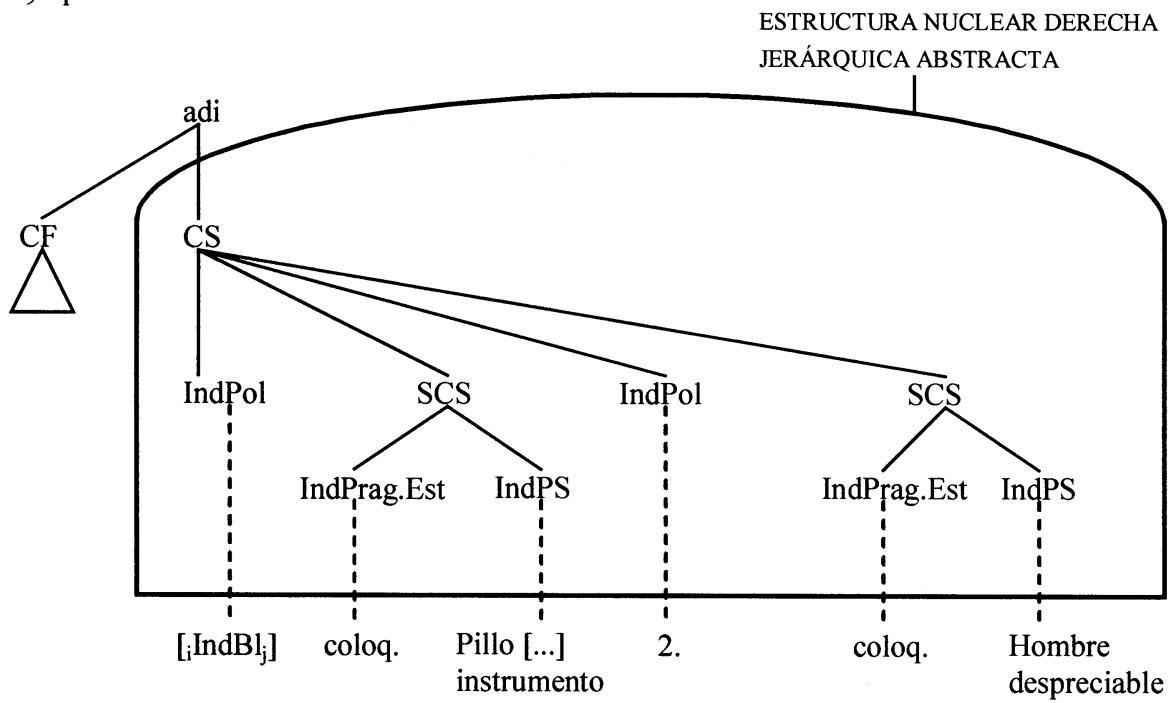

FIGURA 8. Diagrama estructural comentado de la estructura nuclear derecha jerárquica (concreta y abstracta) perteneciente al comentario semántico de adi ${ }_{9}$

pragmáticas repetidas. Sobre todo, se debe fijar explícitamente el alcance de las indicaciones dislocadas. Se le debe explicar al usuario en uno de los metatextos que las indicaciones dislocadas rigen para el uso del signo lemático correspondiente a las paráfrasis de significado siguientes. Esto significa que el alcance textual de las indicaciones del subcomentario semántico dislocado comprende los subcomentarios semánticos que aparecen a continuación. Esto no se desprende automáticamente de las indicaciones dislocadas y de su posición textual, ya que tras la dislocación hacia la izquierda aparecen en el artículo relaciones textuales que no se dan en textos no lexicográficos ${ }^{18}$.

Mientras que en el primer estadio de la condensación textual las operaciones de condensación textual solo afectan a los elementos de los superconjuntos de las microestructuras, la dislocación hacia la izquierda, que constituye el segundo estadio de condensación textual, altera también las relaciones de la estructura.

Este es también el caso en el tercer estadio de la condensación textual interna, del que nos ocuparemos ahora brevemente.

18 Hay otros tipos de dislocación hacia la izquierda de los que no nos podemos ocupar aquí. Nos limitaremos a mencionar que en los artículos de signos lemáticos polisémicos también se puede considerar el comentario sobre la forma en su conjunto como un constituyente textual dislocado hacia la izquierda. 


\subsection{TERCER ESTADIO DE CONDENSACIÓN TEXTUAL INTERNA}

El tercer estadio de la condensación textual interna se alcanza cuando aparecen dislocaciones hacia la derecha que van acompañadas de una condensación textual. Este caso es relativamente poco frecuente. La operación de dislocación hacia la derecha produce inicialmente una relajación textual. Sería erróneo considerar que la relajación textual es siempre lo contrario de la condensación textual, puesto que las operaciones de dislocación hacia la derecha no disminuyen de por sí la densidad proposicional. Más bien, lo que ocurre es simplemente que la distribución de los datos en el artículo se altera de manera que la estructura interna de acceso rápido presenta una mayor diferenciación, con lo que las zonas de búsqueda para determinadas consultas se reducen ( $c f$. Wiegand 1996b).

Un ejemplo de artículo lexicográfico que presenta los resultados de la dislocación hacia la derecha es adi ${ }_{10}$. Aquí, una indicación morfológica ("Morf. sup. irreg. sacratísimo") se ha dislocado hacia la derecha, a la posición inmediatamente posterior a la última indicación de paráfrasis de significado a la que afecta. Al dislocar hacia la derecha esta indicación desde el comentario sobre la forma, la pertenencia a este se pierde en el plano formal del diccionario, por lo que es necesario introducir una indicación en forma de símbolo sobre la pertenencia al comentario sobre la forma, que en este caso es un calderón (“"”) que antecede a la indicación morfológica $^{19}$. Este está direccionado hacia la izquierda, a la indicación sobre la forma del signo lemático. Esta indicación rige para seis subcomentarios semánticos, pero no se repite para cada uno de ellos, sino que se sitúa al final del último para el que es válida. De esta forma, se produce una condensación textual ( $c f$. nota 18).

$\operatorname{adi}_{10}$ : sagrado, da. (Del lat. sacrātus). Adj. Digno de veneración por su carácter divino o por estar relacionado con la divinidad. $\| \mathbf{2}$. Que es objeto de culto por su relación con fuerzas sobre naturales de carácter apartado o desconocido. ॥ 3. Perteneciente o relativo al culto divino. || 4. Digno de veneración y respeto. || 5.inmodificable. Sus costumbres son sagradas. || 6. Entre los antiguos, sobrehumano. $\mid$ MORF. sup. irreg. sacratísimo. || 7. m. Lugar que, por privilegio, podía servir de refugio a los perseguidos por la justicia. U. t. en sent. fig. ॥ acogerse alguien a sagrado. fr. Huir de una dificultad que no puede satisfacer, interponiendo una voz o autoridad respetable. V. cáscara $\sim$, fuego $\sim$, hierba $\sim$, historia $\sim$, letras $\sim$ s, libro $\sim$, Sagrado Texto, vaso $\sim$.

[DRAE-2001]

19 En el prediccionario se proporciona la siguiente explicación sobre las indicaciones dislocadas hacia la derecha: "La información sobre ortografía -especialmente sobre el uso de mayúscula inicial- o sobre determinadas peculiaridades morfológicas de la acepción -superlativos y conjugación de los verbos irregulares en particular-, aparece en un apartado propio, encabezado por la indicación abreviada correspondiente [...]. Si la información afecta a más de una acepción, el mensaje aparece tras la última a la que puede referirse, destacado mediante un calderón (q)" (DRAE, "Condensación textual lexicográfica: esbozo de una concepción integral"). 
Al igual que la dislocación hacia la izquierda, la dislocación hacia la derecha afecta a las relaciones de la microestructura. A partir de artículos con microestructuras sencillas integradas, se llega a artículos que presentan microestructuras parcialmente integradas con un integrado intermedio, como en este caso, o también con un posintegrado (cuando, como consecuencia de la dislocación hacia la derecha, el elemento dislocado pasa a ocupar la última posición), también llamadas microestructuras anexadas ( $c f$. Wiegand 1996b: 9 y ss.).

Tenemos ahora una cierta visión de conjunto de la condensación textual interna, aun sin entrar en los detalles de su diversidad empírica (se pueden encontrar más ejemplos en Wolski 1991 para diccionarios bilingües, en Ranenführer y Böhme 1995 para los diccionarios ortográficos y en Wiegand 1996c).

A continuación nos ocuparemos de la condensación textual externa.

\section{CONDENSACIÓN TEXTUAL EXTERNA}

Los textos lexicográficos que experimentan la condensación textual externa son los textos parciales con una estructura de acceso externa, como, sobre todo, los catálogos de palabras. La condensación textual externa afecta, por tanto, principalmente a los portadores de elementos conductores, es decir, a los elementos de los superconjuntos de las macroestructuras y de las estructuras de acceso externas, y, en consecuencia, principalmente a los lemas y sublemas. La condensación textual externa puede afectar también a la macroarquitectura del catálogo de palabras.

Encontramos un ejemplo de condensación textual externa en $\operatorname{adi}_{11}$ :

$\mathrm{Adi}_{11}$ : Hänge|ärmel mpl mangas fpl perdidas $\|$

-backen $f p l\langle$ fam $\rangle$ mofletes mpl $\|$-bahn $f$

[Seilbahn] ferrocarril aéreo od colgante, teleférico $\mathrm{m} \|$ [Schiene] monocarril $\mathrm{m}$ aéreo $\|$

-balken $m\langle$ Zim $\rangle$ puntal $\mathrm{m}$ pendiente, sopanda $\mathrm{f} \|$

-bank $f\langle\mathrm{Bgb}\rangle$ descargadero, taquete $\mathrm{m} \|$-bauch

$m$ vientre $\mathrm{m}$ colgante od caído $\|$-bett $n$ cama $\mathrm{f}$

colgante $\|$-boden $m\langle$ Arch $\rangle$ techo $\mathrm{m}$ colgante od

suspendido || [zum Wäschetrocknen] secadero $\mathrm{m} \|$

-brücke $f$ puente m colgante od de suspensión \|

-brust $f$ 〈wiss $\rangle$ mama f péndula, 〈fam〉 pechos

mpl caídos $\|$-gerüst $n \cdot$ Arch $\rangle$ andamio $\mathrm{m}$

colgado $\|$-isolator $m\langle\mathrm{El}\rangle$ aislador $\mathrm{m}$ de

suspensión $\|$-lager $n\langle$ Tech $\rangle$ soporte m colgante

od suspendido $\|$-lampe $f$, -leuchter $m$ lámpara $\mathrm{f}$

colgante od de suspensión \| [Krone] araña $\mathrm{f} \|$

-leiter $f$ [beim Turnen] escalera foblicua $\|$ 
-lippe flabio $\mathrm{m}$ be(l)fo, jeta $\mathrm{f} \|$ ? mit $-\mathrm{n}$ be $(l)$ fo,
jetudo

[Slabý-Grossmann-Illig]

La condensación textual consiste aquí en que en los signos lemáticos de compuestos (por ejemplo, Hängebacken) se sustituye el primer constituyente por un guion. Surgen así lemas parciales ( $c f$. Wiegand 1983a: 454, definición 49) que el usuario concreto ha de completar.

Otra forma de condensación textual externa es la que encontramos en los artículos de remisión condensados, como en $\operatorname{adi}_{12}$ :

Adi $_{12}$ : Fondeadero, fondear, fondillos, fondo, fondón, V. hondo

[Corominas]

El artículo de remisión condensado adi ${ }_{12}$, del que se pueden obtener cinco remisiones, se puede interpretar como resultante de cinco artículos de remisión diferentes en sucesión alfabética que sufren un proceso de condensación textual:

$\begin{aligned} & \text { adi }_{13} \text {-adi }_{17} \text { Fondeadero V. hondo } \\ & \text { Fondear V. hondo } \\ & \text { Fondillos V. hondo } \\ & \text { Fondo V. hondo } \\ & \text { Fondón V. hondo }\end{aligned}$

En el paso de los cinco artículos de remisión al artículo de remisión condensado, se omiten, por ejemplo, todas las indicaciones de remisión "V. hondo" menos la última. A continuación, para ahorrar espacio, los lemas restantes se enlazan, con lo que surge $\operatorname{adi}_{12}$.

También el grado de condensación de las remisiones condensadas puede variar. En adi $_{10}$ se encuentran las siguientes remisiones: "V. cáscara $\sim$, fuego $\sim$, hierba $\sim$, historia $\sim$, letras $\sim$ s, libro $\sim$, Sagrado Texto, vaso $\sim$.". Se sustituye aquí el segundo componente de las expresiones pluriverbales por una virgulilla, lo que representa un mayor grado de condensación en comparación con remisiones como las de $\operatorname{adi}_{12}$.

Respecto de la macroestructura, en los artículos de remisión condensados encontramos un aglomerado lemático. En cuanto a la microestructura, en $\operatorname{adi}_{12}$ se encuentra una secuencia de indicaciones sobre la forma del signo lemático y, en las remisiones situadas al final de $\mathrm{adi}_{10}$, una sucesión de indicaciones sobre la forma del signo lemático condensadas a partir de las cuales se puede llegar a $\mathrm{n}$ signos lemáticos $(\mathrm{n}>1)$.

Por último, nos referiremos brevemente a la formación de nichos. La ordenación no alfabética se puede interpretar como un resultado de la condensación textual, lo que queda de manifiesto al compararla con la ordenación alfabética simple. El 
$D R A E$ es un diccionario con ordenación alfabética simple. De él hemos tomado $\operatorname{adi}_{18}-\operatorname{adi}_{30}$ :anticlerical. adj. Contrario al clericalismo. Apl. a pers., u. t. c. s. \|2. Contrario al clero.

anticlericalismo. m. Doctrina o procedimiento contra el clericalismo. \|2. Animosidad contra todo lo que se relaciona con el clero.

anticlímax. m. Gradación retórica descendente. || 2. Término más bajo de esta gradación. || 3. Momento en que descien-de o se relaja la tensión después del clímax.

anticlinal. (Del gr. $\alpha \dot{\nu} \tau \iota \kappa \lambda i ́ v \varepsilon \iota v$, inclinar en sentido contrario). adj. Geol. Se dice del plegamiento de las capas del terreno en forma de A o de V invertida. U. m. c. s. m. V. plegamiento .

anticodón. m. Biol. Secuencia de tres unidades en un ARN transferente que reconoce el codón correspondiente en un ARN mensajero.

anticolegialista. adj. Ur. Opuesto al régimen colegiado de gobierno. U. t. c. s.

anticolonial. adj. Contrario al colonialismo.

anticolonialista. adj. Opuesto al colonialismo. Apl. a pers., u. t. c. s.

anticomunismo. $\mathrm{m}$. Tendencia contraria al comunismo.

anticomunista. adj. Contrario al comunismo. Apl. a pers., u. t. c. s.

anticoncepción. f. Acción y efecto de impedir la concepción.

anticoncepcional. (De anticoncepción). adj. anticonceptivo. U. t. c. s. m. anticoncepcionismo. $\mathrm{m}$. anticoncepción. || 2. Doctrina que propugna el empleo de prácticas para evitar la concepción.

[DRAE-2001]

En los diccionarios que siguen un orden alfabético simple, la disposición de los lemas es vertical y cada artículo lexicográfico abarca exactamente un bloque de texto ( $c f$. Wiegand 1989a: 386 y ss.). Esta es, por tanto, la forma menos densa que puede presentar el catálogo de palabras de un diccionario que sigue un orden alfabético inicial. Por ello, el catálogo de palabras de un diccionario con orden alfabético simple no solo presenta una estructura textual interna jerárquica que representa una estructura de constituyentes textuales ( $c f$., por ejemplo, Wiegand 1995: $470 \mathrm{y}$ ss.), sino también una macroarquitectura, puesto que en el conjunto de todos los artículos lexicográficos se definen relaciones topológicas, concretamente, una relación encima de y una relación debajo de que no se pueden considerar como parte de la estructura de constituyentes.

$\mathrm{Si} \mathrm{a} \mathrm{adi}_{18}-\mathrm{adi}_{30}$ se les aplica el método de condensación textual adjuntar, la sucesión vertical de lemas se convierte en una sucesión encadenada, desaparece la formación de bloques de texto y se pierde la microarquitectura, puesto que los artículos lexicográficos ya no se sitúan unos debajo de otros como textos parciales independientes. 
Así, la formación de nichos ahorra espacio, puesto que la superficie de impresión disponible contiene más caracteres que antes de la formación del nicho. Esta constatación nos permite introducir una diferenciación más.

\section{CONDENSACIÓN TEXTUAL FRENTE A COMPRESIÓN TEXTUAL}

En el modelo de condensación textual lexicográfica en varios estadios que hemos esbozado hasta aquí, aún no se ha tratado explícitamente una diferenciación de la que nos ocuparemos ahora brevemente. Se puede llegar a la condensación textual modificando la proporción de caracteres y contenidos proposicionales de tal manera que aumente la densidad proposicional. Este es el caso de que nos hemos ocupado hasta ahora. Sería un error considerar que este tipo de condensación textual sirve únicamente para ahorrar espacio. Además le permite al usuario experto hallar los datos con mayor rapidez. Sin embargo, existe otro tipo de condensación textual de la que no se puede decir esto último. Esta no tiene que ver con la proporción de caracteres y contenidos proposicionales sino con la proporción de caracteres y espacio de impresión. Este tipo de condensación textual tiene que ver únicamente con el ahorro de espacio. Se consigue, por ejemplo, escogiendo un tipo de letra más pequeño, reduciendo el interlineado y renunciando a señalizar la estructura mediante párrafos, bloques de texto, etc. La formación de nichos pertenece a este tipo condensación textual. Al estudiar la condensación textual lexicográfica resulta útil diferenciar uno y otro tipo terminológicamente. Se puede reservar el término condensación textual para la referida a la proporción de caracteres y contenidos proposicionales, y emplear, en cambio, compresión textual para la referida a la proporción de caracteres y espacio de impresión.

\section{CONCLUSIÓN}

En nuestra opinión, con los instrumentos presentados hasta aquí se puede dar cuenta en su mayor parte de los fenómenos empíricos de condensación textual lexicográfica. Lo que necesita ahora la metalexicografía son estudios empíricos sobre cómo influye la condensación textual lexicográfica en el uso de los diccionarios. Presentan especial interés las indagaciones relativas al problema de dónde se sitúa la frontera entre lo que facilita el manejo y lo que lo dificulta.

\section{LISTA DE ABREVIATURAS}

adi: artículo lexicográfico

CF: comentario sobre la forma

CS: comentario semántico

IndAut: indicación de autor 
IndBL: indicación en blanco

IndClPal: indicación de clase de palabras

IndDoc: indicación de documentación

IndEjCU: indicación de ejemplo de competencia de uso

IndEjDoc: indicación de ejemplo de documentación

IndForSL: indicación sobre la forma del signo lemático

IndFrPl: indicación sobre la frecuencia del plural

IndFuen: indicación de fuente

IndGén: indicación de género

IndGr: indicación gramatical

IndLDoc: indicación de lugar de documentación

IndMorf.Sg: indicación morfológica sobre el singular

IndOrt: indicación ortográfica

IndPg: indicación de página

IndPol: indicación de polisemia

IndPrag.Est: indicación pragmática sobre el estilo

IndPrag: indicación pragmática

IndPragNM: indicación pragmática sobre el carácter no marcado

IndPragSem: indicación pragmático-semántica

IndPS: indicación de paráfrasis de significado

SCS: subcomentario semántico

SCSDIzq: subcomentario semántico dislocado hacia la izquierda

El signo "|" significa simultáneamente

\section{REFERENCIAS BIBLIOGRÁFICAS}

\section{DICCIONARIOS}

[Corominas] = COROMINAS, Joan $\left(1973^{3}\right)$ : Breve diccionario etimológico de la lengua castellana, Madrid, Gredos.

[Corominas-Pascual] = Corominas, Joan y José Antonio PAscual (1980): Diccionario crítico etimológico castellano e hispánico, Madrid, Gredos.

[Covarrubias-Suplemento] = CovarRUBIAS, Sebastián de (2001): Suplemento al tesoro de la lengua castellana, eds. Georgina Dopico y Jacques Lezra, Madrid, Polifemo.

$[D E A]=$ SECO, Manuel y otros (1999): Diccionario del español actual, Madrid, Aguilar.

[DELE $]=$ ALVAR EZQUERRA, Manuel, dir. (1995): Diccionario para la enseñanza de la lengua española, Barcelona-Alcalá de Henares, Biblograf-Universidad de Alcalá de Henares. 
[DRAE-2001] = Real Academia Española (2001): Diccionario de la lengua española, Madrid, Espasa.

[DUEAE $]=$ BatTANer Arias, Paz, dir. (2002): Diccionario de uso del español de América y España, Barcelona, Spes.

[Pagés] = PAGÉs, Aniceto de (s.a.): Gran diccionario de la lengua castellana (de autoridades) con ejemplos de buenos escritores antiguos y modernos, Barcelona, Selección.

[Salamanca $]=$ GuTIÉRREZ CUADRADO, Juan, dir. (1996): Diccionario Salamanca de la lengua española, Madrid, Santillana-Universidad de Salamanca.

[Slabý-Grossmann-Illig] = Slabý, Rudolf J., Rudolf Grossmann y Carlos ILliG $\left(1999^{5}\right)$ : Diccionario de las lenguas española y alemana, Wiesbaden, Brandstetter.

\section{BIBLIOGRAFÍA SECUNDARIA}

AdamziK, Kirsten (1995): Textsorten - Texttypologie. Eine kommentierte Bibliographie, Münster, Nodus (= Studium Sprachwissenschaft 12).

BLÄSI, Christoph et al. (1994): "Lexicographic Standards and Reusability. From metalexicographic Description to a Parsing Procedure", Lexicographica, 10, pp. 221248.

Hellwig, Peter (1984a): "Titulus oder über den Zusammenhang von Titeln und Texten. Titel sind ein Schlüssel zur Textkonstitution", Zeitschrift für germanistische Linguistik, 12, pp. 1-20.

(1984b): "Grundzüge einer Theorie des Textzusammenhangs", en Annely Rothkegel y Barbara Sandig, eds., Text - Textsorten - Semantik. Linguistische Modelle und maschinelle Verfahren, Hamburg, Buske (= Papiere zur Textlinguistik 52), pp. 51-79.

IsING, Gerhard (1978): "Textverdichtung und Redundanz in der Lexikographie", en Wissenschaftliche Zeitschrift der Wilhelm-Pieck-Universität Rostock. Gesellschafts- und sprachwissenschaftliche Reihe 27: 1/2, pp. 9-13.

KAMMERER, Matthias y Herbert Ernst Wiegand (1999): “Über die textuelle Rahmenstruktur von Printwörterbüchern. Präzisierungen und weiterführende Überlegungen", Lexicographica, 14, pp. 224-238.

KAMMERER, Matthias y Werner WOLSKI, eds. (2000): Herbert Ernst Wiegand: Kleine Schriften. Eine Auswahl aus den Jahren 1970 bis 1999 in zwei Bänden. Bd. 1: 1970-1988; Bd. 2: 1988-1999, Berlin-New York, de Gruyter.

Konerding, Klaus-Peter y Herbert Ernst WiEGAND (1994): "Framebasierte Wörterbuchartikel. Zur Systematisierung der lexikographischen Präsentation des Bedeutungswissens zu Substantiven", Lexicographica, 10, pp. 100-170. 
Meyer, Meike y Herbert Ernst Wiegand (2000): "Gemischt-semiintegrierte Mikrostrukturen für deutsch-spanische Printwörterbücher”, en Herbert Ernst Wiegand, ed., Studien zur zweisprachigen Lexikographie mit Deutsch v, Hildesheim-New York, Olms, pp. 87-171 (= Germanistische Linguistik 151-152).

RAHNENFÜHRER, Ilse y Gunnar BÖHME (1995): “Orthographiewörterbuch und Textverdichtung", en Petra Ewald y Karl-Ernst Sommerfeldt, eds., Beiträge zur Schriftlinguistik. Festschrift zum 60. Geburtstag von Dieter Nerius, Frankfurt a.M.Berlin-Bern-New York-Paris-Wien, Lang (= Sprache, System und Tätigkeit 15), pp. 257-268.

WIEGAND, Herbert Ernst (1983a): "Was ist eigentlich ein Lemma? Ein Beitrag zur Theorie der lexikographischen Sprachbeschreibung", en Herbert Ernst Wiegand, ed., Studien zur neuhochdeutschen Lexikographie III, Hildesheim-Zürich-New York, Olms (=Germanistische Linguistik 1-4/82), pp. 401-474.

(1983b): “Ansätze zu einer allgemeinen Theorie der Lexikographie", en Joachim Schildt y Dieter Viehweger, eds., Die Lexikographie von heute und das Wörterbuch von morgen. Analysen - Probleme - Vorschläge, Berlin, Akademie der Wissenschaften der DDR (Linguistische Studien, Reihe A. Arbeitsberichte 109), pp. 92-127.

(1983c): "Überlegungen zu einer Theorie der lexikographischen Sprachbeschreibung", en Karl Hyldgaard-Jensen y Arne Zettersten, eds., Symposium zur Lexikographie. Symposium on Lexicography. Proceedings of the Symposium on Lexicography, September, 1-2 1982, at the University of Copenhagen, Hildesheim-Zürich-New York, Olms (= Germanistische Linguistik 5-6/82), pp. 35-72.

(1984a): "On the Structure and Contents of a General Theory of Lexicography", en R. R. K. Hartmann, ed., LEXeter Proceedings. Papers from the International Conference on Lexicography at Exeter 9-12 September 1983, Tübingen, Niemeyer, (= Lexicographica. Series Maior 1), pp. 13-30.

(1984b): "Prinzipien und Methoden historischer Lexikographie", en Werner Besch et al., eds., Sprachgeschichte. Ein Handbuch zur Geschichte der deutschen Sprache und ihrer Erforschung. 1. Halbbd., Berlin-New York, de Gruyter (= Handbücher zur Sprach- und Kommunikationswissenschaft 2.1), pp. 557620 .

(1985): “Eine neue Auffassung der sog. lexikographischen Definition”, en Karl Hyldgaard-Jensen y Arne Zettersten, eds., Symposium on Lexicography II. Proceedings of the Second International Symposium on Lexicography May 16-17, 1984 at the University of Copenhagen, Tübingen, Niemeyer (= Lexicographica. Series Maior 5), pp. 15-100. 
(1987): "Zur handlungstheoretischen Grundlegung der Wörterbuchbenutzungsforschung", Lexicographica, 3, pp. 178-227.

(1988a): "Vorüberlegungen zur Wörterbuchtypologie. Teil I", en Karl Hyldgaard-Jensen y Arne Zettersten, eds., Symposium on Lexicography III. Proceedings on the Third International Symposium on Lexicography. May, 14-16th 1986 at the University of Copenhagen, Tübingen, Niemeyer (= Lexicographica. Series Maior 19), pp. 3-105.

(1988b): "Wörterbuchartikel als Text”, en Gisela Harras, ed., Wörterbücher. Artikel und Verweisstrukturen. Jahrbuch des Instituts für deutsche Sprache 1987, Düsseldorf, Schwann (= Sprache der Gegenwart LXXIV), pp. 30-120.

(1988c): “'Schanghai bei Nacht'. Auszüge aus einem metalexikographischen Tagebuch zur Arbeit beim Großen Deutsch-Chinesisches Wörterbuch”, en Herbert Ernst Wiegand, ed., Studien zur neuhochdeutschen Lexikographie VI, 2. Teilbd. Mit einem Namen- und Sachregister zu den Bänden I-VI sowie einer Bibliographie zur Wörterbuchforschung, Hildesheim-Zürich-New York, Olms (= Germanistische Linguistik 87-90), pp. 522-626.

(1989a): “Aspekte der Makrostruktur im allgemeinen einsprachigen Wörterbuch: Alphabetische Anordnungsformen und ihre Probleme", en Franz Josef Hausmann et al., eds., Wörterbücher. Ein internationales Handbuch zur Lexikographie, 1. Teilbd., Berlin-New York, de Gruyter (= Handbücher zur Sprachund Kommunikationswissenschaft 5.1), pp. 371-409.

(1989b): "Der Begriff der Mikrostruktur: Geschichte, Probleme, Perspektiven”, en Franz Josef Hausmann et al., eds., Wörterbücher. Ein internationales Handbuch zur Lexikographie, 1. Teilbd., Berlin-New York, de Gruyter (= Handbücher zur Sprach- und Kommunikationswissenschaft 5.1), pp. 409-462.

(1989c): "Arten von Mikrostrukturen im allgemeinen einsprachigen Wörterbuch", en Franz Josef Hausmann et al., eds., Wörterbücher. Ein internationales Handbuch zur Lexikographie, 1. Teilbd., Berlin-New York, de Gruyter (= Handbücher zur Sprach- und Kommunikationswissenschaft 5.1), pp. 462-501.

(1989d): "Die lexikographische Definition im allgemeinen einsprachigen Wörterbuch", en Franz Josef Hausmann et al., eds., Wörterbücher. Ein internationales Handbuch zur Lexikographie. 1. Teilbd., Berlin-New York, de Gruyter (= Handbücher zur Sprach- und Kommunikationswissenschaft 5.1), pp. 530-588.

(1990): "Printed Dictionaries and Their Parts as Texts. An Overview of More Recent Research as an Introduction”, Lexicographica, 6, pp. 1-126.

(1991): "Über die Strukturen der Artikeltexte im 'Frühneuhochdeutschen Wörterbuch'. Zugleich ein Versuch zur Weiterentwicklung einer Theorie lexikographischer Texte", en Ulrich Goebel y Oskar Reichmann, eds., Historical Lexico- 
graphy of the German Language, Lewiston-Queenston-Lampeter, Mellen, pp. 341-673.

(1993): "Kritische Lanze für Fackel-Redensartenwörterbuch. Bericht und Diskussion zu einem Workshop in der österreichischen Akademie der Wissenschaften am 14.2.1994", Lexicographica, 9, pp. 230-271.

(1994): "Deutsch-Türkmenisches Wörterbuch. Einblicke in die Wörterbucharbeit an der Türkmenischen Staatlichen Magtymguly-Universität in Aschghabat", Lexicographica, 10, pp. 249-300.

(1995): "Lexikographische Texte in einsprachigen Lernerwörterbüchern. Kritische Überlegungen anläßlich des Erscheinens von Langenscheidts Großwörterbuch Deutsch als Fremdsprache", en Heidrun Popp, ed., Deutsch als Fremdsprache. An den Quellen eines Faches. Festschrift für Gerhard Helbig zum 65. Geburtstag, München, Iudicium, pp. 463-499.

(1996a): "Über die Mediostrukturen bei gedruckten Wörterbüchern”, en Arne Zettersten y Viggo Hjørnager Pedersen, eds., Symposium on Lexicography VII. Proceedings of the Seventh Symposium on Lexicography May 5-6, 1994 at the University of Copenhagen, Tübingen, Niemeyer (= Lexicographica. Series Maior 76), pp. 11-43.

(1996b): "Das Konzept der semiintegrierten Mikrostrukturen. Ein Beitrag zur Theorie zweisprachiger Printwörterbücher", en Herbert Ernst Wiegand, ed., Wörterbücher in der Diskussion II. Vorträge aus dem Heidelberger Lexikographischen Kolloquium, Tübingen, Niemeyer (= Lexicographica. Series Maior 70), pp. 1-82.

(1996c): "Textual Condensation in Printed Dictionaries. A Theoretical Draft", Lexikos, 6, pp. 133-158.

(1996d): "A theory of lexicographic texts. An overview", South African Journal of Linguistics, 14, pp. 134-149.

(1996e): "Deutsch-Usbekisches Wörterbuch. Einblicke in die Wörterbucharbeit an der Staatlichen Usbekischen Weltsprachen-Universität in Taschkent", Lexicographica, 12, pp. 190-254.

(1997a): "Printed Language Dictionaries and their Standardization: Notes on the Progress Toward a General Theory of Lexicography", en Henrick Hock, ed., Historical, Indo-European, and Lexicographical Studies. A Festschrift for Ladislav Zgusta on the Occasion of his 70th Birthday, Berlin-New York, Mouton de Gruyter (= Trends in Linguistics. Studies and Monographs 90), pp. 319-380.

(1997b): "Über die gesellschaftliche Verantwortung der wissenschaftlichen Lexikographie", Hermes, 18, pp. 177-202. 
(1998a): Wörterbuchforschung. Untersuchungen zur Wörterbuchbenutzung, zur Theorie, Geschichte, Kritik und Automatisierung der Lexikographie. 1. Teilbd., Berlin-New York, de Gruyter.

(1998b): "Lexikographische Textverdichtung. Entwurf zu einer vollständigen Konzeption", en Arne Zettersten y Viggo Hjørnager Pedersen, eds., Symposium on Lexicography VIII. Proceedings of the Eighth Symposium on Lexicography, May 2-4, 1996, Tübingen, Niemeyer [= Lexicographica. Series Mayor 90], pp. $1-35$.

(1999): “Artikel einsprachiger Lernerwörterbücher, Textgestaltwahrnehmung und Suchbereichsstrukturen. Plädoyer für übersichtliche Printwörterbücher im Zeitalter der Neuen Medien”, en Bernd Skibitzke et al., eds., Linguistik und Deutsch als Fremdsprache. Festschrift für Gerhard Helbig zum 70. Geburtstag, Tübingen, Niemeyer, pp. 259-281.

(2000a): "Über Suchbereiche, Suchzonen und ihre textuellen Strukturen in Printwörterbüchern”, en Herbert Ernst Wiegand, ed., Wörterbücher in der Diskussion IV. Vorträge aus dem Heidelberger Lexikographischen Kolloquium, Tübingen, Niemeyer (Lexicographica. Series Maior 100), pp. 233-301.

(2000b): “Altes und Neues zur Mediostruktur in Printwörterbüchern”, Lexicographica, 18, pp. 168-252.

(2001): "Sprachkontaktwörterbücher, Typen, Funktionen, Strukturen”, en Birgit Igla et al., eds., Theoretische und praktische Probleme der Lexikographie. 1. Internationales Kolloquium am Institut Germanicum der St. Kliment-OhridskiUniversität Sofia, 7. bis 8. Juli 2000, Hildesheim-New York, Olms (= Germanistische Linguistik 161-162), pp. 115-224.

(2002a): "Zur Makrostruktur und zu den äußeren Zugriffsstrukturen im de Gruyter Wörterbuch Deutsch als Fremdsprache", en Herbert Ernst Wiegand, ed., Perspektiven der pädagogischen Lexikographie des Deutschen II. Untersuchungen anhand des de Gruyter Wörterbuchs Deutsch als Fremdsprache, Tübingen, Niemeyer (= Lexicographica. Series Maior 110), pp. 413-442.

(2002b): "Über textuelle Strukturen der Wörterbuchartikel und Artikelnischen im de Gruyter Wörterbuch Deutsch als Fremdsprache. Zugleich ein Beitrag zur Weiterentwicklung einer Theorie der Wörterbuchform", en Herbert Ernst Wiegand, ed., Perspektiven der pädagogischen Lexikographie des Deutschen II. Untersuchungen anhand des de Gruyter Wörterbuchs Deutsch als Fremdsprache, Tübingen, Niemeyer, (= Lexicographica. Series Maior 110), pp. 497-595.

(2002c): "Was eigentlich sind Wörterbuchfunktionen? Kritische Anmerkungen zur neueren und neuesten Wörterbuchforschung", Lexicographica, 17, pp. 217248. 
(2004): “Überlegungen zur Typologie von Wörterbuchartikeln in Printwörterbüchern. Ein Beitrag zur Theorie der Wörterbuchform”, Lexicographica, 19, pp. 169-313.

WOLSKI, Werner (1989a): "Das Lemma und die verschiedenen Lemmatypen”, en Franz Josef Hausmann et al., eds., Wörterbücher. Ein internationales Handbuch zur Lexikographie. 1. Teilbd., Berlin-New York, de Gruyter (= Handbücher zur Sprach- und Kommunikationswissenschaft 5.1), pp. 360-371.

_ (1989b): "Formen der Textverdichtung im allgemeinen einsprachigen Wörterbuch", en Franz Josef Hausmann et al., eds., Worterbücher. Ein internationales Handbuch zur Lexikographie. 1. Teilbd., Berlin-New York, de Gruyter (= Handbücher zur Sprach- und Kommunikationswissenschaft 5.1), pp. 956-967.

_ (1991): "Formen der Textverdichtung im zweisprachigen Wörterbuch", en Franz Josef Hausmann et al., eds., Wörterbücher. Ein internationales Handbuch zur Lexikographie. 3. Teilbd., Berlin-New York, de Gruyter (= Handbücher zur Sprach- und Kommunikationswissenschaft 5.3), pp. 2837-2854.

ZAIPING, Pan y Herbert Ernst Wiegand (1995): "Über die Musterartikel für das Große Deutsch-Chinesische Wörterbuch. Zugleich ein Beitrag zu einer Theorie zweisprachiger lexikographischer Texte", en Herbert Ernst Wiegand, ed., Studien zur zweisprachigen Lexikographie mit Deutsch II, Hildesheim-New York, Olms (=Germanistische Linguistik 127-128), pp. 63-190. 